\title{
A Review of 5G Front-End Systems Package Integration
}

\author{
Atom O. Watanabe, Student Member, IEEE, Muhammad Ali, Student Member, IEEE, \\ Sk Yeahia Been Sayeed, Rao R. Tummala, Life Fellow, IEEE, P. Markondeya Raj, Senior Member, IEEE.
}

\begin{abstract}
Increasing data rates, spectrum efficiency and energy efficiency have been driving major advances in the design and hardware integration of $\mathrm{RF}$ communication networks. In order to meet the data rate and efficiency metrics, 5G networks have emerged as a follow-on to $4 \mathrm{G}$, and projected to have $100 \mathrm{X}$ higher wireless date rates and $100 \mathrm{X}$ lower latency than those with current 4G networks. Major challenges arise in the packaging of radio-frequency front-end modules because of the stringent low signal-loss requirements in the millimeter-wave frequency bands, and precision-impedance designs with smaller footprints and thickness. Heterogeneous integration in 3D ultra-thin packages with higher component densities and performance than with the existing 2D packages is needed to realize such $5 \mathrm{G}$ systems. This paper reviews the key building blocks of $5 \mathrm{G}$ systems and the underlying advances in packaging technologies to realize them.
\end{abstract}

Index Terms-5G, Millimeter wave, Heterogeneous Integration, Packaging, Antenna in Package.

\section{Path to $5 \mathrm{G}$}

$\mathbf{M}$ ILLIMETER-wave (mm-wave) telecommunication for fifth-generation $(5 \mathrm{G})$ communication is finally becoming a reality. $5 \mathrm{G}$ will impact our lives more dramatically than any technology shift since the internet itself simply because $5 \mathrm{G}$ leads us to a fully connected world. The realization of $5 \mathrm{G}$ drives new packaging technologies compared to conventional RF packaging. This review paper thoroughly covers key research the advances and their technology integration towards the $5 \mathrm{G} \mathrm{mm}$-wave packaging. It begins with a brief introduction of $5 \mathrm{G}$ systems, applications, and performance attributes for demystifying the need for advanced packaging building blocks (Section II). Section II briefly covers the system architecture perspective of 5G technologies. Section III highlights packagelevel technical challenges, followed by the key technology elements for heterogeneous package integration as the key enabler for $5 \mathrm{G}$ (Section IV). Section $\mathrm{V}$ covers the packaging trends in RF front-end, material development for $5 \mathrm{G}$, integration of passive components, and antenna-integrated packages. This review paper also discusses recent demonstrations of 5G $\mathrm{mm}$-wave packages, followed by the introduction of the potential of sixth-generation $(6 \mathrm{G})$ communications and associated opportunities in packaging (Section VI).

A. O. Watanabe, M. Ali, and R. Tummala are with the Department of Electrical and Computer Engineering, Georgia Institute of Technology, Atlanta, GA, 30332 USA e-mail: atom@gatech.edu.

S. Y. B. Sayeed and P. M. Raj are with the Department of Biomedical Engineering, Florida International University, Miami, FL 33174 USA.

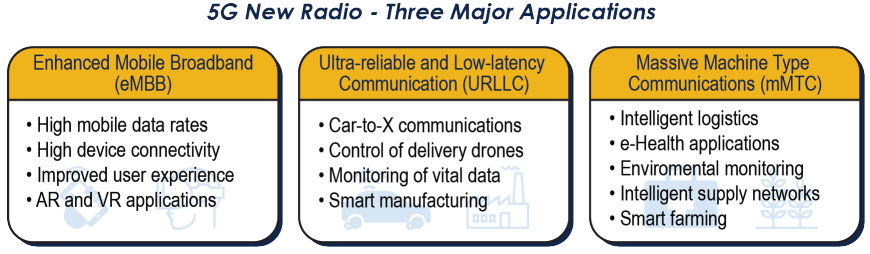

Fig. 1. Major classes of applications with 5G networks.

\section{A. $5 G$ vs $4 G$ Communications}

$5 \mathrm{G}$ is anticipated to be disruptive and life changing as it promises a significantly broader range of applications than its previous counterparts: 4G and long term evolution (LTE), and LTE Advanced (LTE-A/4.5G) networks. Voice was the sole purpose of earlier cellular networks such as $2 \mathrm{G}$ and Enhanced Data Rates for GSM Evolution (EDGE). Design changes subsequently were needed because the high latency of $2 \mathrm{G}$ and EDGE systems was unable to fulfill the need for mobile internet access. This resulted in the dawn of $3 \mathrm{G}$ network, which not only provided more capacity for voice but also introduced fast data services as the network was packaged-switched unlike its circuit switched predecessors. $4 \mathrm{G}$ was evolutionary to $3 \mathrm{G}$ as it increased the capacity by providing higher data rates and added more services at an affordable cost to the consumer [1].

Unlike LTE which is an evolutionary measure, $5 \mathrm{G}$ is an entire new network with a broad range of applications and use-cases [2]. That is why new radio (NR) is the term coined for $5 \mathrm{G}$ as it uses denser modulation schemes, waveforms and pairing with current technologies to meet the ever-growing demands of communication networks. The transition to $5 \mathrm{G}$ from LTE is already much faster than it was for $3 \mathrm{G}$ to $4 \mathrm{G}$ as technologies have rapidly advanced. The users of $5 \mathrm{G}$ go beyond consumer-centric networks and include businesses, services, utilities, cities, and beyond. Speed, capacity, number of connected devices, latency and reliability are the key metrics that are driving the advent of 5G. Given these diverse requirements, the challenge is that $5 \mathrm{G}$ network is expected to support them in a flexible way with high fidelity [3]. Major improvements in 5G compared to current LTE-A networks are listed in Table [

\section{B. 5G Applications (eMBB, URLLC, mMTC)}

Enhanced mobile broadband (eMBB), ultra-reliable low latency communication (URLLC) and massive machine type communication (mMTC) are the three use cases of $5 \mathrm{G}$ air 
interface as shown in Fig. 1. The focus of eMBB is to support ever-increasing demand of end user data rates by increasing system capacity [4]. This is achieved by pushing the envelope of current limitations of the frequency spectrum, the primary being available bandwidth. This limitation is overcome by licensing available bandwidth in sub-10 GHz range as well as opening mm-wave frequencies, where wide bandwidth can be easily allocated. Antenna arrays with multiple antenna elements enable massive multiple input multiple output (MIMO) and beamforming, which multiplies the capacity of a network by exploiting multi-path propagation. eMBB is the initial phase and face of $5 \mathrm{G}$ deployment and it is helping to develop modern broadband use-cases such as Ultra-HD and $360^{\circ}$ streaming, and emerging augmented reality (AR) and virtual reality (VR) media and applications.

The ability to process and harmonize various inputs for fast response falls under the domain of URLLC. It provides ultra response connections with < 1-ms latency and $99.9999 \%$ availability of connection along with support of high-speed mobility for mission critical applications [5]. Some of the usecases of URLLC services include security, vehicle-to-vehicle (V2V) and vehicle-to-everything (V2X) applications, healthcare, utilities, cloud and real-time monitoring situations. The primary advantage of URLLC is not speed but its unfettered reliability to support robust and autonomous real-time decision making [6].

Finally, mMTC service targets robust and cost-sensitive connection of billions of devices such as Internet of Things (IoT) with long-time availability and low power consumption. As the name suggests, it is primarily for machine-to-machine (M2M) applications with minimal human interaction. This service considers IoT with low data rate for a large number of connected devices such as sensors with long range and low maintenance times [7]. Essentially, mMTC entails a large mesh of low-cost, densely connected devices.

\section{C. $5 G$ Frequency Bands}

The 3rd Generation Partnership Project (3GPP) unites seven telecommunications standard development organizational partners in six countries and provides their members with a stable environment to produce the reports and specifications that cover cellular telecommunications technologies. 3GPP has defined two frequency range designations: Frequency Range

TABLE I

IMPROVEMENTS IN 5G TECHNOLOGY COMPARED TO LTE-A

\begin{tabular}{ccc}
\hline Parameter & 5G & $\begin{array}{c}\text { Improvement } \\
\text { compared to LTE-A }\end{array}$ \\
\hline \hline Device/km ${ }^{2}$ & $\begin{array}{c}10^{6} \\
\text { devices/km }\end{array}$ & $10-100 \mathrm{X}$ \\
\hline Latency & $<1 \mathrm{~ms}$ & $10 \mathrm{X}$ \\
\hline Data Rate & $10-20 \mathrm{Gbps}$ & $10-20 \mathrm{X}$ \\
\hline Frequency Range & $600 \mathrm{MHz}$ to $\mathrm{mm}-\mathrm{Wave}$ & $600 \mathrm{MHz}$ to $5.925 \mathrm{GHz}$ \\
\hline $\begin{array}{c}\text { Channel } \\
\text { Bandwidth }\end{array}$ & $100 \mathrm{MHz}$ for $<6 \mathrm{GHz}$ & $5 \mathrm{X}-20 \mathrm{X}$ \\
\hline
\end{tabular}

1 (FR1) and Frequency Range 2 (FR2). FR1 covers lowand mid-frequency bands whereas FR2 is solely for mmwave bands. The frequency range of FR1 and FR2 are defined below:

- FR1: $450 \mathrm{MHz}$ - $6000 \mathrm{MHz}$ (3GPP Rel. 15 V15.4.0 [8]), $410 \mathrm{MHz}$ - 7125 MHz (3GPP Rel. 15 V15.5.0 [9])

- FR2: $24250 \mathrm{MHz}$ - $52600 \mathrm{MHz}$

Till 3GPP Release 15 V15.4.0 [8], the maximum frequency range of FR1 was $6000 \mathrm{MHz}$ hence the name "sub-6 GHz" became a norm but it was changed to $7125 \mathrm{MHz}$ in $3 \mathrm{GPP}$ Release 15 v15.5.0. FR2 has remained the same till the latest report (3GPP Release 16 v16.3.0) [10]. . The 5G spectrum is classified into low-, mid- and high-bands. They are defined below:

- Low-band: $<1 \mathrm{GHz}$

- Mid-band: sub-7 GHz (1 GHz - 7.125 GHz)

- High-band: mm-wave (above $24 \mathrm{GHz}$ )

In the US, Federal Communications Commission (FCC) regulates the communications by radio, television, wire, satellite, and cable. FCC has its own categorization of $5 \mathrm{G}$ spectrum for low-, mid- and high-bands licensed in the US [11].

With the rapid need of $5 \mathrm{G}$ roll-out, two approaches are given by 3GPP: non-standalone (NSA) and standalone (SA). NSA is the early version of $5 \mathrm{G} \mathrm{NR}$ as it uses LTE radio access network (RAN) and core with additional support for 5G lowand mid-bands (sub-7 GHz). This variant is for fast-to-launch approach favored by many carriers throughout the world. On the other hand, the long-term variant, SA has advantages in terms of simplicity and improved efficiency of $5 \mathrm{G}$ next gen core, lower costs, steady improvement of performance in the entire network while enabling URLLC and mMTC use-cases. To be precise, SA gives $5 \mathrm{G}$ NR the ability for independent deployment as it is an end-to-end solution with solid basis to unleash its full potential. However, it has multiple timeconsuming and cost-demanding challenges associated with it such as building new 5G infrastructure [12].

As an inference from the two methods to approach $5 \mathrm{G}$, it can be asserted that NSA $5 \mathrm{G}$ requires more incremental improvements over its SA counterpart. 4G LTE had 40 bands and the 5G cellular networks support even more low- and mid-bands, resulting in complexity in hardware design. The first phase of $5 \mathrm{G}$ deployment and its implementation has introduced challenges by adding more bands in FR1. Similarly, FR2 requires addition of new hardware, resulting in added complexity and miniaturization challenges. This has driven advances in all aspects of the system hardware: from ICs to devices and from cellular devices to base stations. The lowband $5 \mathrm{G}$ using $600 \mathrm{MHz}$ spectrum (band n71) was launched in the US in December 2019 [13-15].

\section{5G SYSTEM DRIVERS}

Systems that operate in $5 \mathrm{G}$ are classified as User Equipment (UE), Customer Premise Equipment (CPE), and Base Station or infrastructure. They have varying needs, size, and power constraints. UE is driven by miniaturization and reduced power while infrastructure equipment is geared towards high gain, communication range, massive-MIMO needs, and broadband. 
TABLE II

TECHNOLOGY METRICS FOR 5G COMMUNICATION SYSTEMS*

\begin{tabular}{|c|c|c|c|}
\hline & $\begin{array}{c}\text { Downlink } \\
\text { (Base station) }\end{array}$ & Uplink (CPE) & User equipment (UE) \\
\hline $\begin{array}{l}\text { Antenna and } \\
\text { module size }\end{array}$ & $70 \times 70 \times 2.7 \mathrm{~mm}^{3}$ & $\begin{array}{c}450-1400 \mathrm{~mm}^{2} \\
\text { Substrate thickness: } 1.5 \mathrm{~mm}\end{array}$ & $20 \times 5 \times 2 \mathrm{~mm}^{3}$ (Qualcomm, QTM052) \\
\hline Antennas & $64-256$ & $16-32$ & $4-8$ \\
\hline PA power & $33 \mathrm{dBm}$ & $19 \mathrm{dBm}$ & 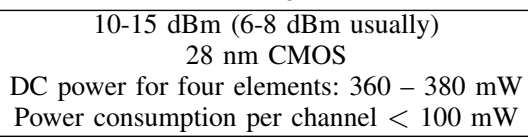 \\
\hline Antenna gain & $\begin{array}{l}27 \mathrm{dBi}^{\mathrm{EIRP}}{ }^{1} \text { of } 50 \\
\mathrm{dBm}(\mathrm{IBM})\end{array}$ & $\begin{array}{c}18-21 \mathrm{dBi} \text { for } 8 \times 4 \text { array patch antenna with } \\
\text { grounded rings }\end{array}$ & $20 \mathrm{dBi}$ for $2 \times 2$ antenna array \\
\hline End-to-End loss & - & - & $2.5 \mathrm{~dB}$ \\
\hline Pathloss & $135 \mathrm{~dB}$ & $135 \mathrm{~dB}$ & - \\
\hline Received power & $-75 \mathrm{dBm}$ & $-90 \mathrm{dBm}$ & $-140 \mathrm{dBm}^{2}$ \\
\hline SNR per RX Element & $5 \mathrm{~dB}$ & $-15 \mathrm{~dB}$ & $6.2 \mathrm{~dB}^{3}$ \\
\hline Rx gain & $21 \mathrm{dBi}$ & $27 \mathrm{dBi}$ & $\sim 10 \mathrm{dBi}$ \\
\hline Rx SNR after gain & $26 \mathrm{~dB}$ & $12 \mathrm{~dB}$ & - \\
\hline \multicolumn{4}{|c|}{$\begin{array}{l}\text { * Parts of this table are taken from }[16] \\
{ }^{1} \text { Effective isotropic radiated power (EIRP) } \\
{ }^{2} \text { Minimum reference signal received power (RSRP) } \\
{ }^{3} \text { Minimum performance requirements for 16-QAM modulation format and } 0.48 \text { code rate for Rank } 1 \text { (beamforming), defined by } 3 \mathrm{GPP} \text { [17 }\end{array}$} \\
\hline
\end{tabular}

The technology metrics for these classes are compared in Table II.

Complexity of 5G systems is a direct result of the addition of features to established hardware, which requires tighter integration, inter-operability, and shielding of all components and devices in a module. Similarly, signals at higher frequencies experience higher losses in free-space due to higher attenuation as predicted by Friis transmission equation. A solution to mitigate this attenuation is to use highly-focused, narrow beams (beamforming and MIMO) to communicate with the target nodes in an efficient manner. This is accomplished by using higher order antenna arrays which are driven by multiple active components. MIMO beamforming technique is primarily classified into three categories: digital, analog, and hybrid. In analog beamforming, amplitude and phase variation is controlled as modulated signals at transmit end, while the received signals from each antenna are summed before the analog-to-digital (ADC) conversion. In digital beamforming, the amplitude/phase variation is controlled as demodulated signals at transmit end while received analog signals are processed after the ADC. Hybrid beamforming technique takes the advantages of both analog and digital beamforming types, which include high flexibility, channel configuration, and low cost and power consumption. The base station can thus serve multiple users in a timeslot.

Analog beamforming is preferred in mobile systems because a single RF chain can address the antenna elements while beam control is performed with passive elements. Digital beamforming consumes higher power with complex hardware but can have full beam control with several simultaneous beams and multiple apertures for future ultra-wide-band communication and software-defined radio features for smart and dynamic spectrum hopping and band-selection. In such innovative digital beamforming approach, the antenna outputs are encoded and multiplexed, and digitized with a single ADC, while demultiplexing and beamforming are performed in the digital domain [18]. Novel onsite coding approach that combines frequency and code multiplexing is developed to reduce the power requirements of digitizer by 10 to $32 \mathrm{X}$ [19]. In order to attain in-band duplex operation to effectively utilize the current-congested spectrum allocation, systems employ time (TDD) or frequency division duplexing (FDD) where the interference between transmit and receive signals is suppressed by differentiating them in time or frequency. However, both require doubling the time or frequency resources. This limitation is addressed with simultaneous transmit and receive (STAR) systems to better utilize the 5G spectrum. In-band full-duplex operation is dependent upon the cancellation of interference between the strong Tx and Rx using techniques such as cross-polarization array elements, and filtering in the analog processing to reduce Tx harmonics, and digital signal processing to filter out multipath domains [20], [21].

The need for low losses demands close integration between antennas with high-power PA and low-noise-figure LNA. As the antenna element size and pitch are comparable to the wavelength, antenna-in-package solutions make this approach feasible with $\mathrm{mm}$-wave communications. Integrating antenna into a packaging substrate reduces the overall interconnect length between RFIC and antennas, and, therefore, mitigates the feed-line loss and enhances the antenna efficiency [22]. In conjunction with the prior popularity of AiP in the $60-\mathrm{GHz}$ band designed for radar, the trend of AiP for $28-\mathrm{GHz}$ or $39-$ $\mathrm{GHz}$ has been more prominent for recent years for consumer electronics.

The choice and integration of TRx technologies vary with the number of antenna elements and antenna gain to attain the required signal power. The radio-frequency front-end (RFFE), control and calibration circuits are integrated into the CMOS [23], SiGe BiCMOS [24], [25] or GaAs [26] transceiver dies, and are connected to the antennas, passive components, and power circuitries using routing layers in the package, microbumps, and through package vias (TPVs). Lower number of elements utilize $\mathrm{GaN}$ while higher antenna elements are driven by SiGe BiCMOS, and much higher antenna elements with 
bulk CMOS. Highly-integrated wireless transceivers further benefit from deep sub-micron to $28 \mathrm{~nm}$ technology to achieve single-chip CMOS solutions.

CMOS and SiGe PAs are optimized for $25 \mathrm{dBm}$ power and $30 \%$ power efficiency while GaAs achieves $30-35 \mathrm{dBm}$ and GaN providing capabilities beyond $45 \mathrm{dBm}$. A 64-element array needs up to $5 \mathrm{~W}$ per PA to reach $240 \mathrm{~W}$. Each PA must operate in the linear regime to reduce the error vector magnitude, which is more challenging for 64-array antennas. As the efficiency of PA modules with the required 8-dB backoff is estimated as $40 \%$, the increased power dissipation from the combined analog and digital processing requires integrated thermal management.

\section{Packaging Challenges in MM-WAVE 5G}

Packaging of 5G systems needs integration of RF, analog and digital functions along with passives and other system components in a single module. These systems ideally exemplify the heterogeneous integration trend. This becomes more important for $5 \mathrm{G}$ because of several reasons: a) integration of antennas with transceiver ICS and associated passive and RF power divider netweorks, b) addition of sub- $6 \mathrm{GHz}$ (FR1) in the short-term with advances in packaging technologies, c) new mm-wave bands (FR2) drive the integration of new filters and diplexers along with broadband power amplifiers and switches, d) the add-on modules to the existing RFFE put additional emphasis on miniaturization and component integration. Proximity of the transceiver and front-end module is also important to reduce the size and losses. This is achieved by integration of antennas with the RF module as well as simultaneous modeling of a heat dissipation solution to keep active components in acceptable thermal conditions. Integration of power amplifiers with antenna arrays needs to address the challenges with size, cost, and performance [27]. These challenges translate to multi-layer fabrication with fine-line features and precise layer-to-layer registration, advanced lowloss materials to reduce conductive losses and co-simulation of circuit, device, package, and thermal solutions. The emerging $3 \mathrm{D}$ package integration solutions also underscore the need for isolation between the various circuit blocks. Because of the deployment of such high-power amplifiers and a large antenna array in millions of base-stations, cost needs to be addressed for high-volume manufacturing.

\section{A. High-density integration of mm-wave components for $5 G$ $R F$ front-end modules}

5G mm-wave modules entail tight integration of antenna arrays, transceiver ICs, power-management ICs, the stack of logic-memory, and surface mounted passive components, as depicted in Fig. 2. The reported thickness of mm-wave packaging substrates varies from $0.15 \mathrm{~mm}$ to $1.2 \mathrm{~mm}$. The variation results mainly from the antenna-in-package requirements. Packages without antenna could employ thin substrates below $300 \mu \mathrm{m}$. However, antenna-integrated modules require higher thickness as antenna arrays offer higher bandwidth with a thicker substrate due to more separation of ground plane from

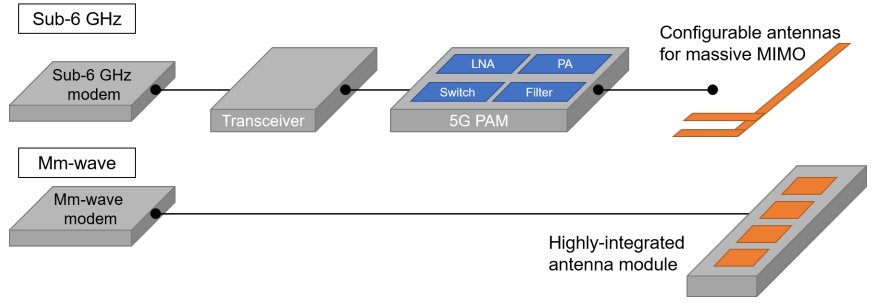

Fig. 2. An example of tight integration in RFFE architectures for 5G electronics.

antenna patches. The thickness of entire modules (packagingsubstrate thickness + mold height) also varies from $0.5 \mathrm{~mm}$ to $2 \mathrm{~mm}$. In addition to the thickness requirement for antenna, the system components and interconnects (Fig. 2) determine the total thickness and area of the entire packages.

\section{B. Novel circuit design techniques}

Integration of multiple components into a package require more complicated signal routing and power delivery network in a limited space, to attain the required signal and power integrity and the control of electromagnetic-interference (EMI). The dielectric thickness for signal routing and power distribution can be $15 \mu \mathrm{m}$ and lower [28].

\section{Multi-physics analysis and modeling}

Ideally, devices, component, and interconnects should maintain stable performance under various signal, power, and thermomechanical loads. As discussed in [29], [30], the packaging structure affects not only the signal and power distribution but also the electrical performance of each component or device. This demands accurate and reliable multi-physics modeling and analyses from the electrical, materials, chemical and mechanical standpoints.

\section{Co-Simulation of package, circuit and device}

5G mm-wave technology, as discussed in Section II. entails co-design of phased antenna array with transceiver or beamforming ICs to meet the power distribution and signal integrity requirements, and passive components to support all the functions with minimal parasitics and interference. The co-design should also consider electromagnetic compatibility and thermal design, as exemplified by $\mathrm{Gu}$ et al. [22].

\section{E. Novel Materials with good electrical and thermal proper- ties}

In conjunction with the traditional material requirements for dielectrics in IC packaging such as compatible CTE and Youngs modulus, the new class of requirements especially for AiP leads to the development of low-loss dielectric materials. Low-loss materials are translated to materials with low dissipation factor, or loss tangent $(\tan \delta)$. Low $\tan \delta$ mitigates dielectric loss in in-package interconnects, feedlines, and antennas, and thus increases the antenna efficiency. The high losses in the power amplifiers make thermal management 
more challenging. The recent notable material development is discussed in Section IV-B. In addition, there are studies on advanced RF conductors other than conventional copper, such as graphene [31], graphene composite [32], copper paste [33], artificial magnetic conductors [34], metasurface [35], and metaconductors [36]. These materials provide lower resistivity, superior impedance control, or lower manufacturing cost over the traditional copper patterning.

\section{F. Process challenges for high precision patterning}

Requirements for RFFE packaging differ from those for high-performance computing. RFFE conventionally prioritizes impedance control more than the high number of $\mathrm{I} / \mathrm{O}$ or the bandwidth of the interconnects. However, emerging heterogeneous-integration trend to support massive MIMO will require finer-pitch I/O between the chip, the package, or antenna array. It is, therefore, imperative to manage both impedance control and minimize tolerances of the manufacturing processes for small line width and spacing (L/S) down to $5 / 5 \mu \mathrm{m}$ to $2 / 2 \mu \mathrm{m}$ by 2025 and $1 / 1 \mu \mathrm{m}$ by 2030 [37]. The emergence of AiP in front-end packages underscores the importance of multi-layer fabrication and layer-to-layer alignment with the precisely-controlled dielectric thickness. The fabrication accuracy of multi-layers is one of the key metrics to obtain good model-to-hardware correlation. Furthermore, metallization quality plays an important role. The surface roughness of the conductor degrades the signal quality, which needs to be incorporated in the process design to mitigate the system interconnect loss in conjunction with the effort to lower the dielectric loss.

\section{G. Thermal management for $5 G$}

Reduced distance and the increased number of component density call for integrated heat-spreading structures from the thermal-management standpoint. $5 \mathrm{G} \mathrm{mm-wave} \mathrm{technology} \mathrm{is}$ expected to consume more power than the previous generations of wireless technologies [38]. Efforts to address thermalmanagement challenges have been made both by the academia [39], [40] and industry [22], [41], [42] mostly with electrical and thermal co-design and analysis.

\section{5G Packaging Technology Building Blocks}

\section{A. Packaging Trends for $5 G$ Systems}

System-level packaging in the mm-wave technology are partitioned into baseband modules and antenna-integrated transceiver modules. In such packages, interconnections between ICs and other elements such as antenna, passive components, and PCBs must satisfy several requirements. One of the most critical requirements is the impedance control especially in the analog domain. In the mm-wave antennain-package solutions, interconnections between transceiver ICs and antennas should result in low insertion loss and acceptable return loss over the frequency range of interest. The other key requirement is the form factor; two types of interconnect techniques are widely available in the packaging industry, and a third technique has been rapidly emerging during the past

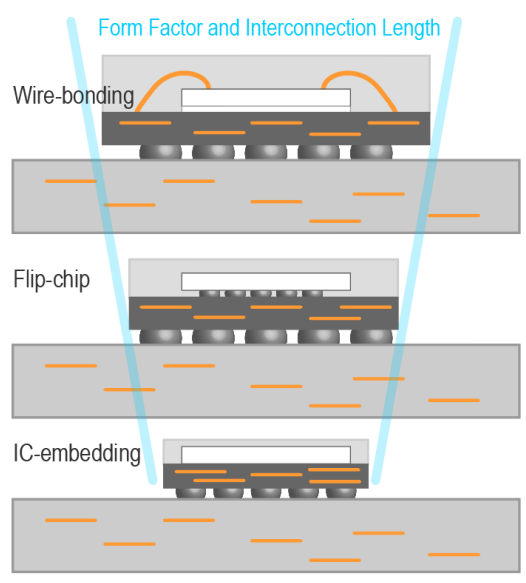

Fig. 3. Trends of interconnection assembly methods in RF/mm-wave technology for miniaturization. Wire-bonding as the most traditional low-I/O interconnection, flip-chip interconnection down to $25 \mu \mathrm{m}$, and IC-embedding or fan-out interconnection.

decade. The two conventional techniques are wire-bonding (Fig. 33) and flip-chip interconnections (Fig. 3p), whereas the emerging technique is referred to as IC-embedding or fan-out packaging (Fig. 33). Although flip-chip and fan-out interconnections have originally been developed for highperformance computing (HPC) or mobile processor applications, their interconnection attributes such as fine pitch and low electrical parasitics are getting critical in $\mathrm{RF} / \mathrm{mm}$-wave packages such as baseband modules and antenna-integrated modules.

Despite the maturity and cost effectiveness in the packaging industry, interconnection using the wire-bonding technique has been identified as one of the key challenges in systemon-package (SoP) because of the significant signal loss and impedance discontinuity caused by the bond wire, which degrades the performance of the $\mathrm{RF} / \mathrm{mm}$-wave system chain [43]. Interconnection using the flip-chip technique offers better performance than wire-bonding as the bump height is smaller than the length of bond wires. The flip-chip technique is also preferable for small form factor, which provides more than 800 input/output (I/O). The flip-chip technique started from solder bumps with a diameter range of 75-200 $\mu \mathrm{m}$, while copperpillar interconnection with solder cap nowadays reaches down to the diameter less than $40 \mu \mathrm{m}$. The copper-pillar technique not only provides high-density I/O, but also does it offer lower conductive loss.

The evolution of IC-embedding or fan-out packaging has been recently imperative. Embedded wafer-level ball grid array (eWLB) pioneered by Infineon and fan-out waferlevel packaging (FOWLP) are drawing attention as mm-wave packages. This embedding technology eliminates the use of wire bonding which not only introduces significant highfrequency loss and parasitics, but also increases the footprint for high-pin count die. Transceiver ICs are embedded in a reconfigured molded wafer with compression molding process. Multiple redistribution layers (RDLs) are formed to fan-out the baseband signals, and through-mold vias are utilized for vertical interconnections. From the industry standpoint, Infineon, 
(a) Modem Module Package

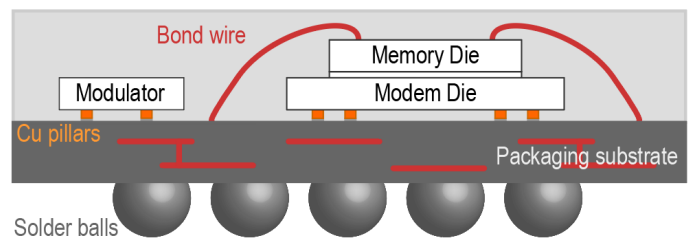

(b) Antenna-Integrated Package

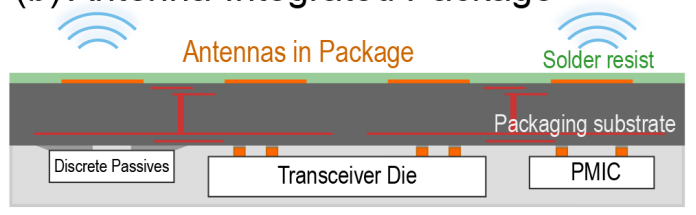

Fig. 4. Examples of system-integrated mm-wave packages (a) modem SoP (b) antenna-integrated package.

TSMC, ASE, Amkor, Deca Technologies, JCET, and several more companies have been leading the wafer-level fan-out technology for RF/mm-wave applications. In conjunction with the molding-compound-based waver-level IC-embedding technology, laminate-based and glass-based panel-level embedding technology is also emerging as an alternative candidate, mainly led by Samsung, Unimicron, ASE, and Deca Technologies from the industry, and Institute of Microelectronics (IME) [44] and Georgia Tech Packaging Research Center (GT-PRC) from the research standpoint [28], [45].

Fig. 4 illustrates the key modem and antenna-integrated packages. Most cases are such modules employ the combination of multiple techniques from the three interconnection methods. Bond wires could serve as interconnection between the PCB board and memory that is stacked atop the logic or modem die, while the modem die with high pin count entails flip-chip interconnects to provide clean signal and mitigated signal delay. The most popular assembly method to integrate a mm-wave phased antenna array with ICs interconnected is, as of now, the flip-chip technique because of the process cost and supply-chain maturity. Conductive materials are selected from copper pillar or $\mathrm{C} 4$ bumps, depending on the pin count and sensitivity of assembled dies to the conductive loss caused by interconnections.

\section{B. Materials for Core, Prepreg, and Buildup}

The selection of dielectric materials is critical to obtain the desired electrical performance of ICs and antenna arrays integrated in 5G module packages. A wide variety of dielectric materials are studied and investigated to meet requirements for various applications such as handset devices, radar modules, base-stations, and satellites. Well-designed dielectric materials provide improved link budget, low signal dissipation, high signal or power density, desired frequency response of passive components, small footprint of elements, lower module thickness, high antenna efficiency and EIRP, beam width, angular coverage, energy consumption, and miniaturization of antennas.
LTCC has been employed for high-frequency antenna modules such as WiGig, alternatively known as $60 \mathrm{GHz} \mathrm{WiFi}$, which includes IEEE 802.11ad standard and also the upcoming IEEE 802.11ay standard. The large number of metal layers and relatively-low $\tan \delta$ are the major advantages of LTCC, as indicated in Table III] Its low coefficient of thermal expansion (CTE) and high thermal conductivity also lead to high reliability and robustness to temperature variations. The major challenges are the low density of signal routings in internal layers and size scalability. As LTCC substrates are fabricated with screen-printing and co-firing processes, the features of RDL are generally large $(>125 \mu \mathrm{m})$ due to the dimensional limit of screen-printing masks and alignment accuracy between layers. This large feature size results in low density of signal routing and entails high number of metal layers, resulting in modules.

The mainstream platform for antenna integrated packages is still based on multi-layered organic substrates. The process for the multi-layered organic substrates for mm-wave modules is similar to that of PCB manufacturing and is cost-effective due to the compatibility with the existing supply chain and highvolume manufacturing for consumer electronics. The organic materials are designed to show lower $\tan \delta$, compared to the traditional FR4, where $\tan \delta$ is higher than 0.02 in the mmwave frequency bands. The mainstream copper-clad laminates (CCL) and prepregs used for formation of multi-layered organic substrates comprises of four classes of polymers: 1) Bismaleimide Triazine (BT), 2) Polyphenyleneether (PPE), 3) Liquid-crystal Polymer (LCP), and 4) Polytetrafluoroethylene (PTFE), as listed in Table III. Unlike glass-cloth epoxy resin (e.g., FR4), glass-cloth PPE substrates feature high glasstransition temperature ( $\mathrm{Tg}$ ), low water absorption, low dielectric constant (both Dk and Df). PPE-based substrates are typified by MEGTRON6 (core and prepreg) from Panasonic, and CS-3376C from Risho Kogyo. Similar to PPE resin,

TABLE III

LOW-LOSS DIELECTRIC MATERIALS RECENTLY USED FOR 5G SUBSTRATE TECHNOLOGY

\begin{tabular}{|c|c|c|c|c|}
\hline & Materials & $\begin{array}{c}\text { Dk } \\
\text { Df }\left(x^{-4}\right)\end{array}$ & $\begin{array}{l}\text { Reported } \\
\text { frequency }\end{array}$ & $\begin{array}{c}\text { Major } \\
\text { suppliers }\end{array}$ \\
\hline \multirow{4}{*}{ 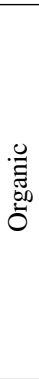 } & $\begin{array}{l}\text { Bismaleimide } \\
\text { Triazine } \\
\text { (BT) }\end{array}$ & $\begin{array}{c}3.4 \\
40 \quad 50\end{array}$ & $10 \mathrm{GHz}$ & Mitsubishi \\
\hline & $\begin{array}{l}\text { Polyphenyl- } \\
\text { ethers } \\
\text { (PPE) }\end{array}$ & $\begin{array}{cc}3.25 & 3.4 \\
20 & 50\end{array}$ & $150 \mathrm{GHz}$ & $\begin{array}{c}\text { Panasonic, } \\
\text { Risho Kogyo }\end{array}$ \\
\hline & $\begin{array}{l}\text { Liquid-crystal } \\
\text { Polymer } \\
\text { (LCP) }\end{array}$ & $\begin{array}{l}2.9 \\
25\end{array}$ & $10 \mathrm{GHz}$ & $\begin{array}{l}\text { Rogers, } \\
\text { Murata }\end{array}$ \\
\hline & $\begin{array}{l}\text { Polytetrafluoro- } \\
\text { ethylene } \\
\text { (PTFE) }\end{array}$ & $\begin{array}{c}2.2 \\
9\end{array}$ & $10 \mathrm{GHz}$ & $\begin{array}{l}\text { Rogers, } \\
\text { DuPont }\end{array}$ \\
\hline \multirow{3}{*}{ 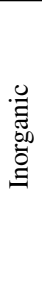 } & $\begin{array}{l}\text { Low-temp. } \\
\text { cofired ceramic } \\
\text { (LTCC) }\end{array}$ & $\begin{array}{c}6 \\
18\end{array}$ & $60 \mathrm{GHz}$ & $\begin{array}{l}\text { Hitachi Metals, } \\
\text { Kyocera, TDK }\end{array}$ \\
\hline & $\begin{array}{c}\text { Borosilicate } \\
\text { glass }\end{array}$ & $\begin{array}{c}5.4 \\
50 \quad 90\end{array}$ & $1060 \mathrm{GHz}$ & $\begin{array}{c}\text { AGC, } \\
\text { Corning, Schott, } \\
\text { 3DGS, NSG }\end{array}$ \\
\hline & Fused silica & $\begin{array}{l}3.8 \\
3 \quad 4\end{array}$ & $1060 \mathrm{GHz}$ & $\begin{array}{c}\text { AGC, } \\
\text { Corning, Schott, } \\
\text { 3DGS, NSG }\end{array}$ \\
\hline
\end{tabular}


the lamination of BT-based core with build-up dielectrics form the multi-layered organic substrates. BT-based laminates provide low CTE, low shrinkage, and high peel strength with copper. LCP and PTFE are gaining attention because of low $\tan \delta$ at the mm-wave frequency range. Unlike PPE and BT, these materials require high-temperature and highpressure processes for lamination and thermal compression. Halogenated substrates are not usually preferred for handset applications for environmental and safety concerns.

The mechanical and process limitations of organic laminates from their low modulus and high CTE are addressed with emerging inorganic substrates, as listed in Table III Glass substrates offer a wide range of Dk $(3.7-8)$ and Df ( 0.0003 for fused silica to 0.006 for alkaline-free borosilicate), smooth surface, good dimensional stability $(<2 \mu \mathrm{m}$ for 20 $\mathrm{mm}$ substrates), large-panel scalability, ability to form finepitch through vias, stability to temperature and humidity, and tailorable CTE depending on the packaged components [28], [46-49]. Companies such as Samtec and Unimicron manufacture glass-based packages. However, glass-based packaging still poses challenges associated with process immaturity and higher cost resulting from lack of the supply-chain readiness and the nature of glass such as brittleness or robustness and difficulty in handling.

In conjunction with the substrate materials, the formation of RDL and microvias [50] becomes more critical to form high-density interconnects $(<10 \mu \mathrm{m})$ and to enable tight integration of components. Dry-film dielectric materials show good compatibility with panel-level packages with double-side RDLs and offer a wide thickness variation $(>5 \mu \mathrm{m})$. Lowloss or low $\tan \delta$ materials are developed in the industry for recent mm-wave packages, as shown in Table IV. Low-loss dry-films, which are typically epoxy-based, usually contain silica or ceramic fillers to lower the dissipation factor $(\tan \delta)$. The fillers increase Youngs modulus, decrease CTE, and lead to technical challenges such as the narrow process window, poor adhesion to substrate materials, and reliability in harsh environment. The other method to form dielectric is the use of liquid-based dielectric such as polyimides. As opposed to dry films, liquid-based dielectric materials are more compatible with wafer processes or build-up layers on one side of the substrate. Non-filler photo-sensitive or photo-imageable lowloss liquid-based dielectric materials designed for $5 \mathrm{G} \mathrm{mm}$ wave applications are emerging to form fine features below 5 $\mu \mathrm{m}$ [51-53]. Low-loss liquid-based dielectric materials from major suppliers are listed in Table V. These materials are, however, not still in high volume manufacturing phase. The other challenge is that these materials are spin-coated or slitcoated and have difficulty in forming films with thickness more than $40 \mu \mathrm{m}$.

\section{Integration of Passive Components}

Passive components play a key role in wireless system implementations as there is a need to provide matching impedances for components such as PAs and LNAs, filtering, tuning and biasing [54]. Passive components also make up functions such as couplers, baluns, power combiners and
TABLE IV

LOW-LOSS BUILD-UP DRY FILMS FOR HIGH-DENSITY SIGNAL ROUTINGS FOR HIGH-FREQUENCY APPLICATIONS

\begin{tabular}{cccccc}
\hline & Dk & $\begin{array}{c}\text { Df } \\
\left(\times 10^{-4}\right)\end{array}$ & Frequency & $\begin{array}{c}\text { CTE } \\
(\mathrm{ppm} / \mathrm{K})\end{array}$ & $\begin{array}{c}T_{g} \\
\left({ }^{\circ} \mathrm{C}\right)\end{array}$ \\
\hline \hline Ajinomoto & 3.3 & 44 & $5.8 \mathrm{GHz}$ & 20 & 153 \\
DOW & 2.57 & 32 & $1 \mathrm{MHz}$ & 63 & 250 \\
Hitachi Chem. & 3.3 & 34 & $5 \mathrm{GHz}$ & 17 & 233 \\
Sekisui & 3.3 & 37 & $5.8 \mathrm{GHz}$ & 27 & 183 \\
Taiyo Ink & 3.3 & 2530 & $560 \mathrm{GHz}$ & 20 & 160 \\
\hline
\end{tabular}

TABLE V

LOW-LOSS PHOTOSENSITIVE (PHOTO-IMAGABLE) DIELECTRICS FOR HIGH-DENSITY SIGNAL ROUTINGS FOR HIGH-FREQUENCY APPLICATIONS

\begin{tabular}{cccccc}
\hline & Dk & $\begin{array}{c}\text { Df } \\
\left(\times 10^{-4}\right)\end{array}$ & Frequency & $\begin{array}{c}\text { Min. } \\
\text { L/S }\end{array}$ & $\begin{array}{c}\text { Elonga- } \\
\text { tion (\%) }\end{array}$ \\
\hline \hline DOW & 2.65 & 8 & $<20 \mathrm{GHz}$ & $18 \mu \mathrm{m}$ & 8 \\
Hitachi Chem. & 2.4 & 18 & $10 \mathrm{GHz}$ & - & - \\
JSR & 2.6 & 48 & $<40 \mathrm{GHz}$ & $8 \mu \mathrm{m}$ & $>50$ \\
Toray & 2.9 & 30 & $1 \mathrm{GHz}$ & 30 & \\
\hline
\end{tabular}

dividers, filters, phase shifters, circulators and isolators. Duplexers are usually paired with RF ICs for their nominal operation on the system level. It is typically estimated that passive components account for $90 \%$ of the component count, $80 \%$ of the size and $70 \%$ of the cost [55].

Discrete Lumped Circuits for sub-6 GHz $5 G$ bands: The evolution of passive components started with low temperature co-fired ceramic (LTCC), which became very popular as the surface-mount technology (SMT) components for resistors, inductors and capacitors. A comparison of five different substrate technologies for passive components in terms of performance, thickness, size and some other parameters is given in Fig. 5 [56]. Moreover, a comparison of filter performance (Q-factor) vs. footprint is given in Fig. 6. Acoustic wave technologies have surpassed the typical lumped and distributed LC networks in sub-6 GHz range in realizing high performance filters, resonators, oscillators and delay lines, and have found many applications in $4 \mathrm{G}$ and LTE networks [57].

Distributed Components for mm-wave: Over the past few decades, several theoretical advancements have been made in the design and fabrication of planar passive components such as filters, power dividers, couplers and baluns with their evolution in terms of reduction in size while improving performance metrics [58]. Some of the transmission lines of choice are microstrip, conductor-backed coplanar waveguide (CBCPW) and substrate-integrated waveguide (SIW). The

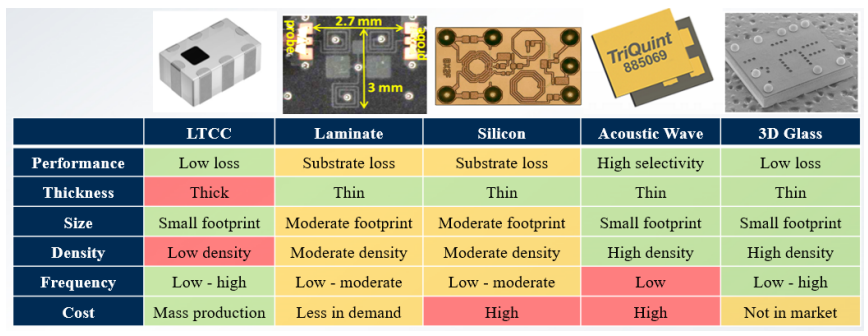

Fig. 5. Evolution of passive component technologies (Courtesy of Zihan Wu, Finisar Corporation) 


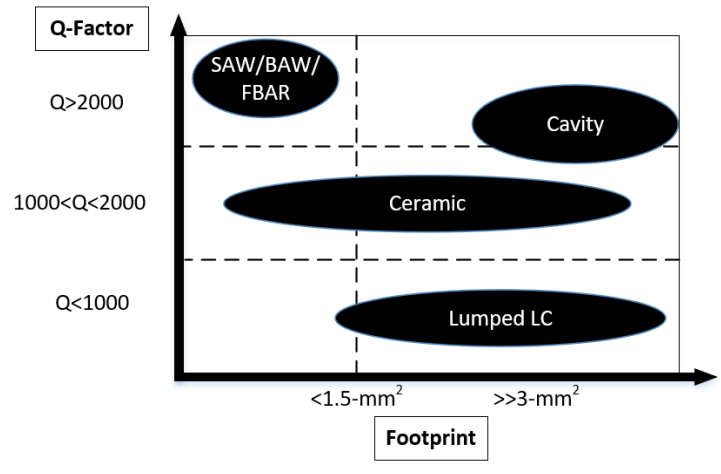

Fig. 6. Comparison of various filter technologies at $1 \mathrm{GHz}$ in terms of performance (Q-factor) and footprint)

technologies include LTCC, organic laminates, state-of-theart integrated fan-out wafer-level packaging (InFO WLP) and ultra-thin laminated glass for passive components realization. A brief review of prior art of these components is this section.

LTCC facilitated initial advances in mm-wave distributed components because of its ability to integrate complex 3D multilayered conductor patterns with through and blind vias. A 4-pole dual-mode resonator filter on LTCC for $30 \mathrm{GHz}$ center frequency with FBW of $4.67 \%$ and an insertion loss of $2.95 \mathrm{~dB}$ using two transmission zeros is reported on a five-metal layer stackup in [59]. Similarly, two-pole, twostage SIW single cavity filter with embedded planar resonators realized on five layers of LTCC with center frequency of 28.12 $\mathrm{GHz}, 15 \% \mathrm{FBW}$ and $0.53 \mathrm{~dB}$ insertion loss is reported for $5 \mathrm{G}$ applications in [60]. A four-pole, four-cavity SIW filter with $2.66 \mathrm{~dB}$ insertion loss at the center frequency of $27.45 \mathrm{GHz}$ with $3.6 \%$ FBW is designed on nine layers of LTCC in [61]. $60 \mathrm{GHz}$ band filters have also been of interest in academia using LTCC stackups [62].

Although LTCC became a very attractive option for fabricating microwave and $\mathrm{mm}$-wave passive components for a couple of decades, challenges such as complexity and cost of fabrication due to high number of layers, loss at mm-wave frequencies and surface roughness led to developments in the wake of relatively new planar organic substrates with stable electrical properties and ease of fabrication. Chen et al., have reported Ka-band four-pole bandpass filters on SIW having asymmetric frequency response for diplexer applications for

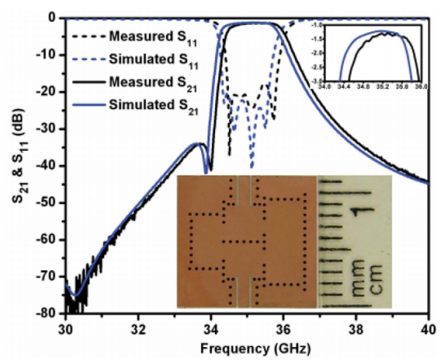

(a)

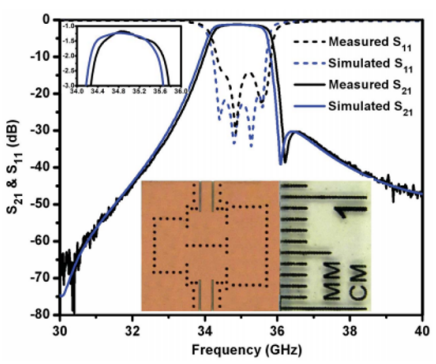

(b)
Fig. 7. Ka-band 4-pole SIW filter on organic laminate (a) TZ on left of passband, and (b) TZ on right of passband 63]

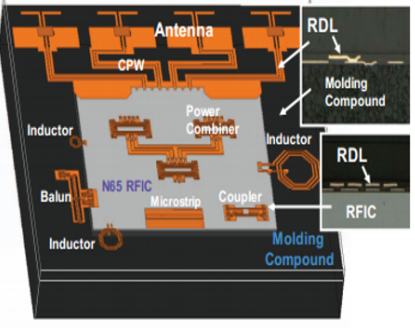

(a)

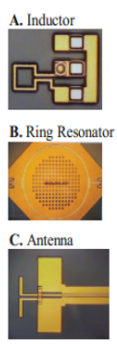

Fig. 8. (a) Schematic of mm-wave circuit including RFIC, passive devices and antennas (b) realized passive devices $[66$

high rejection between neighboring channels [63]. The filters are fabricated on Rogers RT/Duroid 6002 substrate with thickness of $0.508 \mathrm{~mm}$ by using a low-cost PCB process and have transmission zeros (TZ) on either side of the passband as shown in Fig. 7. The center frequency of these filters is 35 $\mathrm{GHz}$ with $3.7 \% \mathrm{FBW}$ and insertion loss of $1.25 \mathrm{~dB}$. Similar filter structures using ridge gap waveguide (RGW) for 30.5 $\mathrm{GHz}$ center frequency are demonstrated by Sorkherizi et al. in [64]. Another research focused on microstrip coupled-line bandpass filters with an insertion loss of $4 \mathrm{~dB}$ and $4.67 \%$ FBW centered at $27.85 \mathrm{GHz}$ on Teflon whereas the filter fabricated on Alumina shows $3 \mathrm{~dB}$ insertion loss and 7.8\% FBW centered at $38.5 \mathrm{GHz}$ [65]. Tsai et al. at TSMC have realized high-performance passive devices for $\mathrm{mm}$-wave system including inductor, ring resonator, power combiner, coupler, balun, transmission line and antennas using InFO WLP [66]. The inductors have a $\mathrm{Q}$ factor of over 40 and the power combiner, coupler and balun show lower transmission loss than on-chip passives. The schematic and fabricated passive devices are shown in Fig. 8

Beyond filters, extensive work is also reported on integrated power dividers for 5G antenna-array applications. A key innovation in these power dividers lies in performance breakthroughs, physical configurations and functional integration with wafer fan-out packaging. Transmission line losses of 0.35 and $0.34 \mathrm{~dB} / \mathrm{mm}$ are achieved with CPW and microstrip loss respectively. One such advance presented by Hsu et $a l$. is an equal-split ultra-miniaturized $\left(960 \times 360 \mu \mathrm{m}^{2}\right) \mathrm{CPW}$ power divider on integrated fanout ( $\mathrm{InFO}$ ) redistribution layers (RDL), with excellent ground shielding capability along with other passive components such as balun and coupler [67]. The power divider exhibits a $10-\mathrm{dB}$ return loss from DC-67 $\mathrm{GHz}$

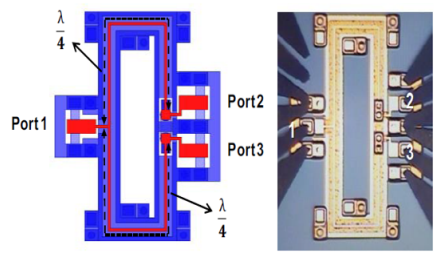

(a)

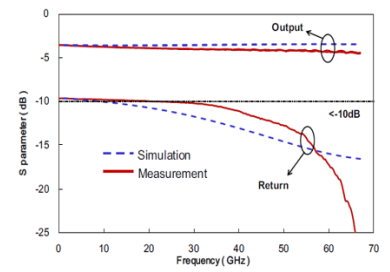

(b)
Fig. 9. CPW power divider on InFO RDL (a) design schematic and fabricated layout, and (b) s-parameters 67 


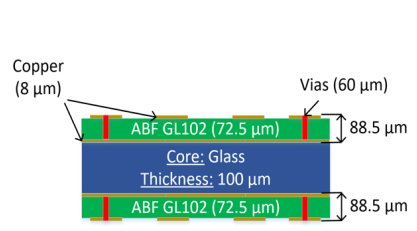

(a)

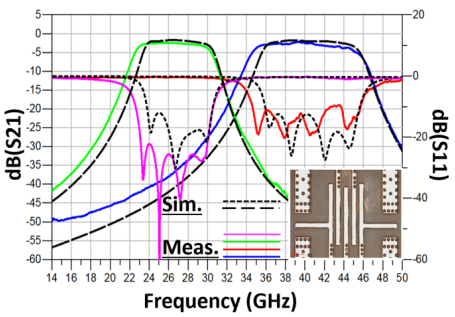

(b)

Fig. 10. (a) Ultra-thin laminated glass-based stackup for 5G filters, (b) sparameters of 5G 28 and $39 \mathrm{GHz}$ interdigital filters and a fabricated interdigital filter coupon [68], [69]

with insertion loss of $4.3 \mathrm{~dB}$ from $30-67 \mathrm{GHz}$. The layout of the power divider with its s-parameters is shown in Fig. 9

Various miniaturized, high performance passive components have been demonstrated on glass [70]. GT-PRC has developed key building blocks such as interconnects, antennas and filters using laminated glass substrate. Test vehicles with six metal layers with the semi-additive patterning (SAP) process enables higher precision on RDL, less than $2 \%$ variance in dimensions and insertion loss of $0.1 \mathrm{~dB} / \mathrm{mm}$ with $15 \mu \mathrm{m}$ buildup dielectric and $0.05 \mathrm{~dB} / \mathrm{mm}$ with $75 \mu \mathrm{m}$ dielectric [28]. Package-integrated and ultra-thin lowpass and bandpass filters with footprint smaller than $0.5 \lambda_{0} \times 0.5 \lambda_{0}$ at the operating frequencies of 28 and $39 \mathrm{GHz}$ bands are developed for $5 \mathrm{G}$ and $\mathrm{mm}$-wave small-cell applications as shown in Fig. 10 [68], [69]. Such package integration of 5G filters with ultra-short 3D interconnects allow for low interconnect losses that are similar to that of on-chip filters, but low component insertion loss of off-chip discrete filters. These thin-film filters exhibit a cross-sectional height of less than $200 \mu \mathrm{m}$ and can be utilized either as embedded components or IPDs in module packages and they can be configured as diplexers as well [71]. A variety of filter structures based on different substrate technologies are compared in Table VI.

In the discussion above, the passive components and their integration is only discussed from the perspective of published research. Component manufacturers have advanced discrete passive components such as filters, couplers and dividers. A

TABLE VI

COMPARISON OF FILTERS USING VARIOUS SUBSTRATE TECHNOLOGIES FOR 5G MM-WAVE APPLICATIONS

\begin{tabular}{|c|c|c|c|c|c|c|}
\hline Ref. & $\begin{array}{c}\text { Substrate Technology / } \\
\text { Structure }\end{array}$ & Order & $\begin{array}{c}\text { IL } \\
(\mathbf{d B})\end{array}$ & $\begin{array}{c}\mathbf{f}_{\mathbf{c}} \\
(\mathbf{G H z})\end{array}$ & $\begin{array}{c}\text { BW } \\
(\mathbf{G H z})\end{array}$ & $\begin{array}{c}\text { Size } \\
\left(\mathbf{m m}^{2}\right)\end{array}$ \\
\hline 60. & LTCC / SIW & 2 & 0.53 & 28.12 & 4.29 & 33.5 \\
\hline 61$]$ & LTCC / SIW & 4 & 2.66 & 27.45 & 0.98 & 43.5 \\
\hline 59] & LTCC / Cavity & 4 & 2.95 & 30 & 1.4 & 88 \\
\hline 63. & Rogers Laminate / SIW & 4 & 1.25 & 35 & 1.3 & 121 \\
\hline 65 & Alumina / Microstrip & 3 & 3 & 38.5 & 3 & 7.1 \\
\hline 72 & $\begin{array}{l}\text { Rogers Laminate/ } \\
\text { Air-filled SIW }\end{array}$ & 4 & 3.9 & 21 & 0.23 & 746 \\
\hline 69$]$ & Glass / Microstrip & 5 & 2.6 & 27 & 5 & 6.9 \\
\hline 69$]$ & Glass / Microstrip & 5 & 1.43 & 40.25 & 6.5 & 5.5 \\
\hline
\end{tabular}

TABLE VII

COMPARISON OF COMMERCIAL FILTERS FOR 5G MM-WAVE APPLICATIONS

\begin{tabular}{cccccc}
\hline Company \& Ref. & $\begin{array}{c}\text { IL } \\
(\mathbf{d B})\end{array}$ & $\begin{array}{c}\mathbf{f}_{\mathbf{c}} \\
(\mathbf{G H z})\end{array}$ & $\begin{array}{c}\text { BW } \\
(\mathbf{G H z})\end{array}$ & $\begin{array}{c}\text { Size } \\
(\mathbf{m m})\end{array}$ \\
\hline \hline TDK & & 1 & 28.5 & 2 & 5 \\
\hline Knowles (band n257) & 74 & 3.5 & 28 & 3 & 12.65 \\
\hline Knowles (band n258) & 75 & 2.9 & 25.875 & 3.25 & 12.65 \\
\hline Knowles (band n260) & 76 & 2.5 & 38.5 & 3 & 14.19 \\
\hline Pasternack $^{*}$ |77 & & 2.5 & 29.25 & 3.5 & 416.7 \\
\hline
\end{tabular}

*9-section bandpass filter.

comparison of various $5 \mathrm{G}$ filters from industry is given in Table VII

\section{Antenna Systems in Package (AiP)}

The 5G wireless communication system entails highlyintegrated radio access solutions, incorporating advanced phased-array antenna and transceiver front-end technology to support high radiated power, large signal-to-noise ratio, as well as beamforming, scanning in elevation and azimuth directions within a wide range [22], [78]. As the antenna element size and pitch are correlated with the wavelength, antenna-in-package solutions become more feasible at $\mathrm{mm}$ wave frequencies unlike discrete antennas for 4G/LTE. Major package-integrated antenna structures are patch antennas and dipole antennas. More number of antenna elements $(N)$ results in additional gain $(10 \log N)$. A $2 \times 2$ antenna array in Qualcomm QTM052 is reported to have a gain of $20 \mathrm{dBi}$, which comprises of $5 \mathrm{dBi}$ per element, $6 \mathrm{dBi}$ from summation, $6 \mathrm{dBi}$ from array formation and additional $3 \mathrm{dBi}$ gain from single polarization.

Patch antennas feature the main lobe in the elevation direction and enable dual-polarization which increases the channel capacity. The size of a patch-antenna array is approximately $\lambda / 2$ (effective half wavelength on dielectric) with a pitch of $\lambda_{0} / 2$ (half-wavelength in air). These fundamental design rules lead to the requirement of high-Dk materials for miniaturization. High-Dk materials, however, degrade the bandwidth and gain. The thickness or separation of the patch and ground is critical since the antenna bandwidth increases almost linearly with the thickness. Another technique to increase bandwidth is the stacked-patch antenna. The stacked patches create two resonances at different frequencies, which also calls for high accuracy of layer-to-layer alignment to obtain desired frequency responses. Antenna designs are performed considering the trade-off between the size and performance (i.e., bandwidth and gain). Common feeding methods are the via-feed or aperture-coupling feed. Via-feeding is relatively simple and guarantees the accuracy of feeding points into the antenna patch. However, the challenge is the limited bandwidth because of the inductive reactance caused by the via [79]. Conformal via shielding is employed to emulate coaxial feed to reduce the undesired inductance and enable better impedance matching. In contrast, aperture-coupled feed 
leads to higher bandwidth than the via feed, while the layerto-layer alignment is extremely critical; a few-micron shift between layers causes the frequency shift and may result in not covering the targeted frequency bands.

Dipole antennas are employed for covering the azimuth direction with a single polarization. Since the length of the dipole is nearly $\lambda_{0} / 2$, the design process is relatively simple. Dipole antennas generally offer wide bandwidths, compared to patch antennas. The gain is controlled by placing additional number of directors in a modified antenna topology referred to as Yagi-Uda antenna. Dipole antenna and YagiUda antenna are fed by two differential transmission lines to reverse the phases of the provided signals. Co-planarwaveguide and stripline signal routing are more common in multi-layered packages because of their inherent shielding features and minimal cross-talk with other nearby transmission lines, components, and antennas.

There are several options to implement mm-wave antenna array, as shown in Fig. 11. The first option refers to implementing antennas directly on the PCB board (Fig. 117) [80-82]. The most notable advantage is the lower cost than the other options because of the supply-chain maturity. The major challenges of antenna on PCB are manufacturing process and tolerances. The relatively-coarse design rules do not allow designers to layout fine structures in transmission-line widths, spaces, via diameters and pitch, and accurate layer-to-layer alignment. Fig. $11 \mathrm{p}$ illustrates the most viable option for $5 \mathrm{G} \mathrm{mm}$-wave applications, the implementation of AiP. In contrast, Fig. 11 . shows the direct antenna implementation onto the IC wafer. Although the antenna-on-chip approach offers the lowest feedline loss and parasitics and provides direct integration with other front-end circuitry [83], the challenges include antenna efficiency, process scalability for large array, yield and cost, thermomechanical reliability issues, and design flexibility.

(a) Antenna on PCB

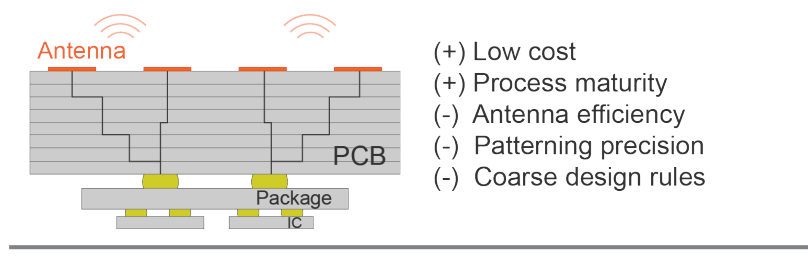

(b) Antenna in Package

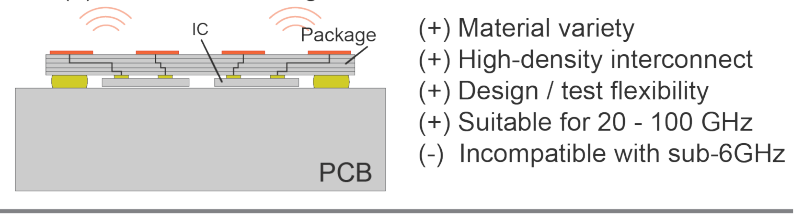

(c) Antenna on Chip

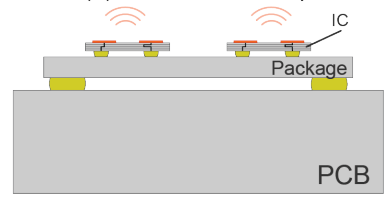

(+) Direct feed from IC

(+) Suitable for $>100 \mathrm{GHz}$

(-) Process cost for array

(-) Thermomechanical reliability

Fig. 11. Three approaches for mm-wave antenna implementation. (a) Antennas on PCB, (b) antennas in package, and (c) antennas on chip or wafer. (a) Flip-chip-based AiP

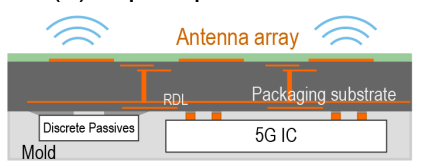

(b) IC-embedded AiP

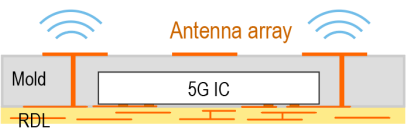

Fig. 12. Packaging structures for antenna integration (a) currently-viable flipchip-based configuration (b) IC-embedded configuration.

\section{Current Development in Heterogeneous PACKAGE INTEGRATION FOR 5G}

This section introduces heterogeneous package integration for mm-wave antenna-integrated packages. Recent mm-wave antenna-integrated packages are classified into two categories based on interconnection techniques: a) chip-last or flip-chip structures, as shown in Fig. 12 and b) chip-embedded structures exemplified in Fig. 12p. System-level architectures and applications are discussed along with the packaging structures and materials in Section IV-B.

IBM made pioneering advances in AiP with organic laminate substrates for base stations, as shown in Fig. 13. The multi-chip antenna-in-package includes 64-array embedded antennas with dual-polarized operation in Tx and Rx modes, with four transceiver ICs that are assembled on the backside with flip-chip technology. In addition, to ensure thermal management, a heat sink is added to ball-grid array (BGA) interface, which allowed to realize consistent board-level assembly. This module operates at $28 \mathrm{GHz}$ and achieves more than $50 \mathrm{dBm}$ EIRP in Tx mode and $\pm 40^{\circ}$ scanning range with $70 \times 70 \times 2.7 \mathrm{~mm}^{3}$ [22], [86]. Ericsson developed similar AiP to implement a reference design for base stations. UCSD also reported 64 dual-polarized dual-beam single-aperture $28-\mathrm{GHz}$ phased array for 5G MIMO, which is implemented in low-cost organic laminates. A $2 \times 4$ dual-beam former chip is flip-chip

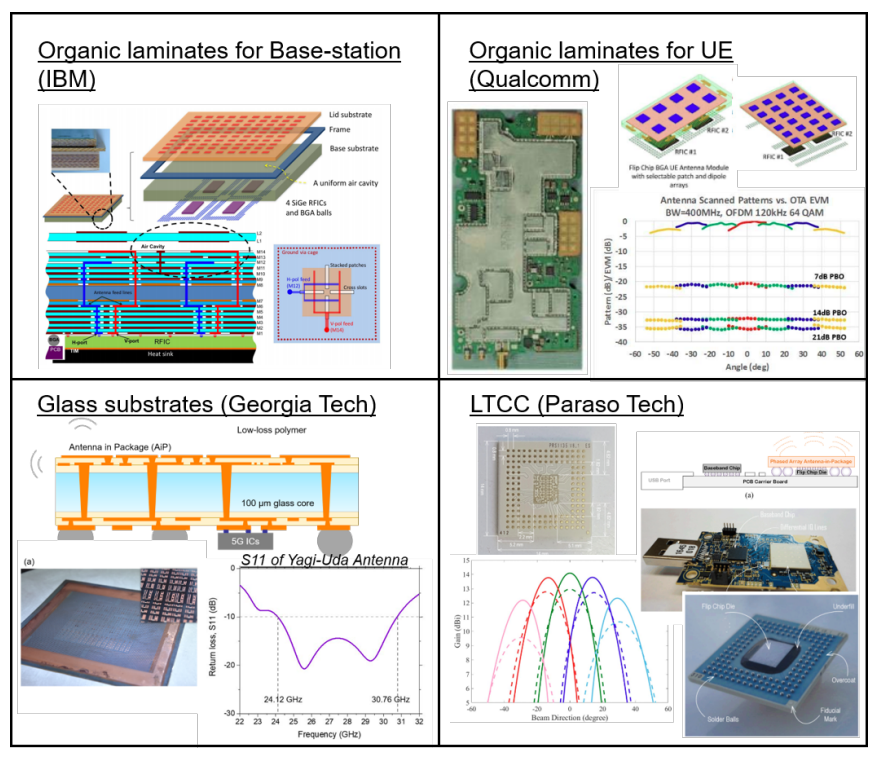

Fig. 13. Demonstrated mm-wave modules with flip-chip-based AiP: organic laminates from IBM [22] and Qualcomm [84], glass-based substrates [28], and LTCC $[85]$. 
assembled on the other side of the antenna array. The array achieved an EIRP of $52 \mathrm{dBm}$ at $29 \mathrm{GHz}$ and scanned $\pm 50^{\circ}$ and $\pm 25^{\circ}$ in azimuth and elevation planes, respectively, with cross-polarization rejection $>30 \mathrm{~dB}$ [87].

Intel [82] has reported a stack-up that consists of an organic laminate-prepreg core for antenna implementation, trace routing, a flip-chip RFIC module that is responsible to quad-feed the antenna where each port is responsible for one band and polarization, and BGA for connection to the main PCB, along with conformal shielding. The package size is $30 \times 15 \times 1.5$ $\mathrm{mm}^{3}$, with 1500 connections for each transceiver IC feeding an $8 \times 4$ patch antenna arras to support linear dual-polarization with $20 \mathrm{~dB}$ isolation and dual-frequency antennas. This work includes the validation of the design and packaging concept suitable for low-cost $5 \mathrm{G} \mathrm{mm-wave} \mathrm{CPE} \mathrm{and} \mathrm{base-station}$ applications. Qualcomm has been pioneering 5G UE modules with ICs in pair with $1 \times 4$ dipole, $1 \times 4$ patch, $2 \times 2$ patch, and $2 \times 4$ patch antenna arrays [84]. The second generation of their antenna SiP module covered dual-band (28 and 39 $\mathrm{GHz}$ ) operation with the size of $19.1 \times 4.9 \times 1.78 \mathrm{~mm}^{3}$. The antenna module includes power-module IC and transceiver IC, which are flip-chip assembled on the other side of the antenna patterns with copper pillars.

As one of the earlier demonstrations for AiP, a $60-\mathrm{GHz}$ antenna integration with transceiver chips in a multi-layer LTCC were achieved by NEC Corporation, IBM [91], [92], Samsung [93], Intel [94], and a few more companies. Recently, Peraso Technologies Inc. in conjunction with Hitachi Metals Ltd. [85] introduced novel corrugated soft or high-impedance surfaces and implemented them between phased-array antenna elements in LTCC procedure for $5 \mathrm{G} \mathrm{mm-wave} \mathrm{communica-}$ tions. Their array preserves $14 \mathrm{dBi}$ gain with 8 elements and $16.5 \mathrm{dBi}$ gain with 16 elements over a $10-\mathrm{GHz}$ bandwidth around $60 \mathrm{GHz}$. Glass-based AiP is also widely investigated especially in recent years [28], [49], [95], [96], because of its potential of electrical properties, dimensional stability, and panel scalability toward $500 \times 500 \mathrm{~mm}^{2}$.

For the next-generation AiP, chip-embedded structures are promising for form-factor and thickness reduction with shorter

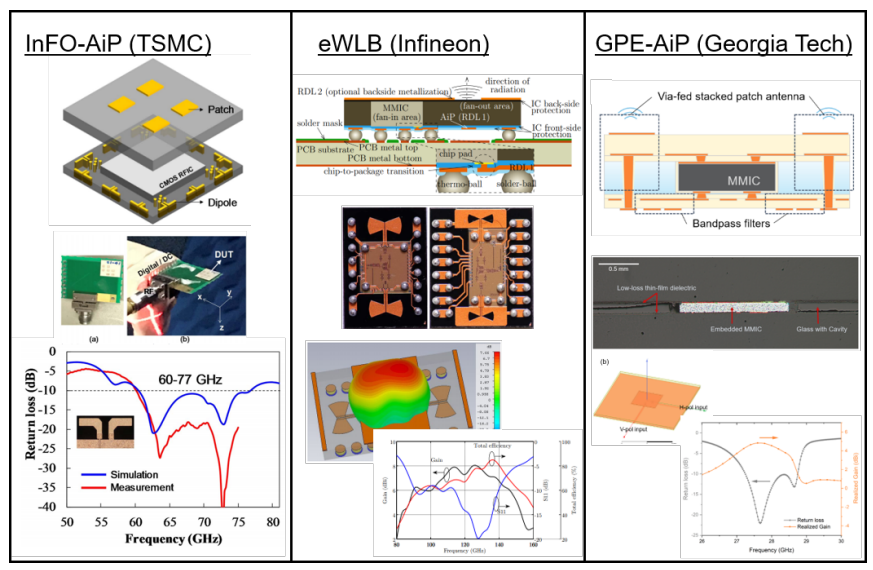

Fig. 14. Demonstrated mm-wave modules with IC-embedded AiP: moldingcompound-based InFO-AiP from TSMC [88], eWLB from Infineon [89], and glass-based [90] fan-out or chip-embedded packaging structures. interconnects, as shown in Fig. 14. As discussed in Section IV-A. Wojnowski and Bock from Infineon, for the first time, reported the fan-out wafer-level packaging (FOWLP) technology with their eWLB and presented antenna-integrated package in 2012 and 2015 [97], [98]. Advanced Semiconductor Engineering (ASE) reported, in 2019, the FOWLP processes for stacked-patch antennas to enhance the bandwidth [99]. TSMC has reported InFO-based antenna-integrated packages with beamforming capability of the antenna array system with $6 \mathrm{dBi}$ gain in a 40-nm CMOS RFIC co-designed system [88]. Tsai et al. reported an antenna-integrated wafer-level package with a size of $10 \times 10 \times 0.5 \mathrm{~mm}^{3}$, which shows an antenna-array gain of $14.7 \mathrm{dBi}$ at the $60 \mathrm{GHz}$ band [100]. In addition to the wafer-level packaging, several R\&D teams from industry and academic research groups are investigating the potential of fanout panel-level packaging (FOPLP) with organic substrates or glass substrates [90], [101] for 5G and radar communications.

\section{5G AND BEYOND - 6G}

$6 \mathrm{G}$ is expected to utilize even higher frequencies and channel bandwidths than $5 \mathrm{G}$, primarily by operating in the terahertz $(\mathrm{THz})$ gap frequency range, resulting in massive data rates of 100 Gbps - 1 Tbps, compared to $10-20$ Gbps of 5G [103]. The research in the domain of sub- $\mathrm{THz}$ to evaluate applications and use-cases is already underway in many focused research groups throughout the world [102], [104]. 5G, as discussed in this paper, is known for its revolutionary flexibility. However, 6G will likely be known for using artificial intelligence (AI) by capitalizing on the backbone of flexibility offered by $5 \mathrm{G}$. The other two use-cases are also expected to significantly advance with the advent of $6 \mathrm{G}$ : massive URLLC to provide true microsecond latency and mMTC to connect hundred of billions of devices. Some of the predicted 6G key performance indicators (KPI) compared to $5 \mathrm{G}$ are shown in Fig. 15 A capability factor of $10-100$ is expected for $6 \mathrm{G}$ as compared to 5G. The key technologies to enable $6 \mathrm{G}$ are AI, advanced RF, optical and network technologies [105], [106]. As a result, we can expect dominance of pervasive AI and machine learning to create smart, self-configurable mesh networks to support scalability with minimal human interaction. With $\mathrm{THz}$ radios,

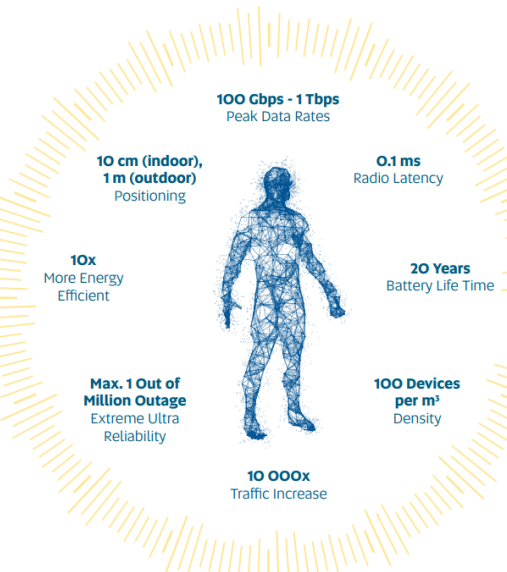

Fig. 15. 6G KPIs compared to $5 \mathrm{G}[102]$. 


\section{G System-on-Package}

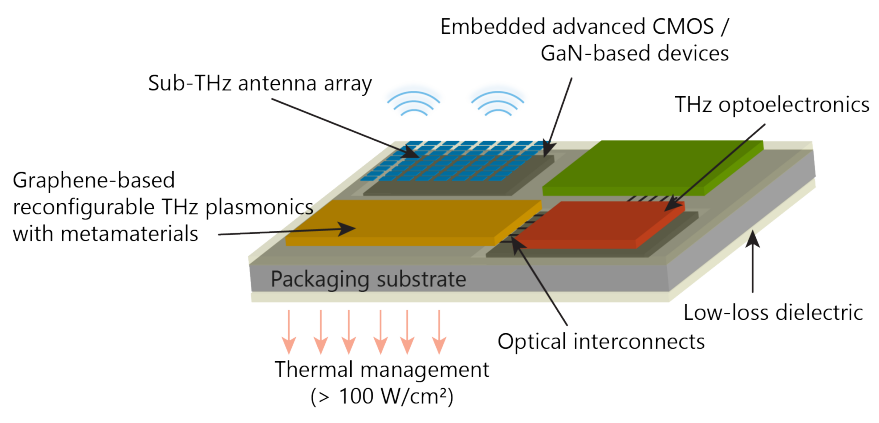

Fig. 16. Conceptual diagram of heterogeneously-integrated quasi-optical $\mathrm{THz}$ package for $6 \mathrm{G}$ communications.

smart sensors and IoT driven by requirements such as HD artificial /virtual reality (AR/VR), extended reality (XR), local processing (wireless $\mathrm{AI}$ ) and others, it would be imperative to identify potential new technologies to realize it, support evolution of use-cases and identify new ones to provide a ubiquitous, ultra-low latency, high-fidelity network regardless of the nature of environment [107], [108].

Many of the traditional approaches used for RF packages are not applicable to sub- $\mathrm{THz}$ and $\mathrm{THz}$ radios. Sub- $\mathrm{THz}$ or $6 \mathrm{G}$ communications focus on heterogeneous package integration, as illustrated in Fig. 16 by incrementally advancing the system components such as precision antenna arrays, low-loss interconnects and waveguides and active devices. As frequency increases into the $\mathrm{THz}$ region, we will not be able to employ the techniques used for low frequencies because of multiple challenges associated with signal loss, dimensions, and materials. The current interconnection techniques such as wire bonding or solder-based bumps are too bulky; therefore, excitation of multimode, radiation, and reflection would adversely affect the electrical performance. As the skin depth is approximately $120 \mathrm{~nm}$ at $300 \mathrm{GHz}$, higher frequency packaging will require shorter interconnects and smooth surface to mitigate conductor loss. Radiation loss must be minimized as well with good impedance matching and high-precision manufacturing with tolerance below $1 \mu \mathrm{m}$. Dielectric loss is more predominant in sub- $\mathrm{THz}$ or $\mathrm{THz}$ bands, which translates to the need for accurate characterization of potential materials at those high frequencies. Another key metric in $\mathrm{THz}$ applications is antenna implementation. While AiP approach is viable, antennas or radiators on ICs will play a significant role. The integrated antenna may include graphene-based radiation sources and detectors, THz antenna arrays (e.g., 1024 elements in $1 \mathrm{~mm}^{2}$ ), lenses, and intelligent metasurfaces with space-time-frequency coding and low-loss interconnects. Fig. 16 shows a conceptual diagram of heterogeneously-integrated quasi-optical $\mathrm{THz} 6 \mathrm{G}$ package for a device with an antenna, where sub- $\mathrm{THz}$ or $\mathrm{THz}$ waves will be radiated into the air from reconfigurable AiP or the on-chip antenna [109]. Technical challenges such as heat management and EMI shielding must be also addressed for such high-frequency systems.

\section{SUMmarY}

Millimeter wave and THz-enabled sub-systems will dominate future communication networks for broadband wireless mobile connectivity, latency-sensitive applications such as vehicle-to-vehicle and vehicle-to-network, and massive connectivity needs in IoT. They will also create mm-wave imaging, sensing, and other applications that simultaneously demand high bandwidth, reliability and zero perceived latency. The associated product segments are classified into basestation, customer premise equipment and user equipment. Despite the wide range of power $(<1 \mathrm{~W}$ to $30 \mathrm{~W})$, communication range (few meters to 100s of meters) and bandwidths requirements, underlying technologies have several similarities in the package architectures, materials and processes. The key challenges with $\mathrm{mm}$ wave communications, such as large path losses, interconnect losses, power-hungry devices, and other technology limits have been systematically addressed with advances in III-V devices, IC-package co-design, innovative beamforming architectures, and test methodologies, making $5 \mathrm{G}$ systems a reality today. The fundamental technologies are systematically classified and reviewed in this paper.

Unlike with 4G, antenna arrays need to be integrated in the 3D package with smaller sizes making antenna integration as the mainstream 5G package architecture. Multilayer organic (MLO) packages with transceiver dies flip-chip-attached to the backside of the package and antenna arrays on the top side, known as AiP phased-array system is widely adapted in todays UE and base station products. Ultra-low transmission losses, below $0.1 \mathrm{~dB} / \mathrm{mm}$, fine lines and spaces with $1-5 \%$ process variations to meet stringent in-band loss and outof-band rejection specifications has been the key packaging focus for $5 \mathrm{G}$ modules. Various passive elements such as filters, power dividers and other functions are embedded into the packages as distributed components. Organic packages, however, are limited in line-width control due to dimensional instability, requiring more layers with low process precision and high via-transition losses, resulting in thicker packages. This has been paving way to inorganic substrates such as glass as an alternative to meet the density and impedance continuities across the packages.

$\mathrm{THz}$ or $6 \mathrm{G}$ communications are the emerging as the next frontier with 100-1000X increase in network speed compared to $4 \mathrm{G}$ and LTE, leading to new applications based on virtual reality, and intense high-resolution video communications and connectivity of a much larger number of devices, such as sensors for IoT. Compact and high-output power sources and high-responsivity low-noise detectors that can operate at $\mathrm{THz}$ band are required to surmount the high path-loss at these frequencies. Key innovations such as integration of graphenebased radiation sources and detectors, $\mathrm{THz}$ antenna arrays, lenses and intelligent meta-surfaces with space-time-frequency coding and low-loss interconnects will create the next set of $R \& D$ challenges for academia and industry.

\section{REFERENCES}

[1] "Evolution of Mobile Technologies," Qualcomm, White Paper, 2014. [Online]. Available: t.ly/HoGT 
[2] "Introducing 5G technology and networks (definition, use cases and rollout)," Thales Group, Tech. Rep. [Online]. Available: https://www.thalesgroup.com/en/markets/ digital-identity-and-security/mobile/inspired/5G

[3] "5G use cases and requirements," Nokia, White Paper. [Online]. Available: $t .1 y / 1 \mathrm{Qbl}$

[4] G. Brown, "Exploring 5G New Radio: Use Cases, Capabilities and Timeline," Heaving Reading - Qualcomm, White Paper, 2016. [Online]. Available: ht.ly/H27V

[5] - "Ultra-Reliable Low-Latency 5G for Industrial Automation," Heaving Reading - Qualcomm, White Paper, 2018. [Online]. Available: https://www.qualcomm.com/media/documents/files/ read-the-white-paper-by-heavy-reading.pdf

[6] M. Bennis, M. Debbah, and H. V. Poor, "Ultrareliable and low-latency wireless communication: Tail, risk, and scale," Proceedings of the IEEE, vol. 106, no. 10, pp. 1834-1853, 2018.

[7] D. Schnaufer, T. Nguyen, B. Thomas, A. Mariani, P. Cooper, B. Peterson, and P. Warder, "5G RF - 2nd Qorvo Special Edition," e-Book, 2017. [Online]. Available: https://www.qorvo.com/design-hub/ ebooks/5g-rf-for-dummies

[8] 3GPP, "5G; NR; Base Station (BS) radio transmission and reception," 3rd Generation Partnership Project (3GPP), Technical Specification (TS) 38.104 Release 15 Version 15.4.0, December 2018. [Online]. Available: http://www.3gpp.org/ftp//Specs/archive/38_series/ 38.104/38104-f40.zip

[9] - "5G; NR; Base Station (BS) radio transmission and reception," 3rd Generation Partnership Project (3GPP), Technical Specification (TS) 38.104 Release 15 Version 15.5.0, May 2019. [Online]. Available: http://www.3gpp.org/ftp//Specs/archive/38_series/ 38.104/38104-f50.zip

[10] - " "5G; NR; Base Station (BS) radio transmission and reception," 3rd Generation Partnership Project (3GPP), Technical Specification (TS) 38.104 Release 16 Version 16.3.0, April 2020. [Online]. Available: http://www.3gpp.org/ftp//Specs/archive/38_series/ 38.104/38104-g30.zip

[11] "5G FAST Plan," FCC, Tech. Rep. [Online]. Available: https: //docs.fcc.gov/public/attachments/DOC-354326A1.pdf

[12] "Making 5G NR a Commercial Reality," Qualcomm, Tech. Rep., February 2020. [Online]. Available: https://www.qualcomm.com/ media/documents/files/making-5g-nr-a-commercial-reality.pdf

[13] T-Mobile, "T-Mobile 5G: Its On! Americas First Nationwide 5G Network Is Here," December 2019. [Online]. Available: https://www.t-mobile.com/news/americas-first-nationwide-5g-network

[14] Samsung, "Samsung Galaxy S10 5G," March 2019. [Online]. Available: https://www.samsung.com/global/galaxy/galaxy-s10/specs/

[15] _ _ "Samsung Galaxy S20 5G," February 2020. [Online]. Available: https://www.samsung.com/us/mobile/galaxy-s20-5g/specs/

[16] T. Cameron, "Bits to Beams-RF Technology Evolution for 5G mmwave Radios," Analog Devices, 2019.

[17] 3GPP, "NR; User Equipment (UE) radio transmission and reception; Part 4: Performance requirements," 3rd Generation Partnership Project (3GPP), Technical Specification (TS) 38.101-4 Release 16 Version 16.0.0, March 2020. [Online]. Available: http://www.3gpp.org/ftp/ /Specs/archive/38_series/38.101-4/38101-4-g00.zip

[18] S. B. Venkatakrishnan, A. Akhiyat, E. A. Alwan, and J. L. Volakis, "Multiband and Multibeam Direction of Arrival Estimation Using On-Site Coding Digital Beamformer," IEEE Antennas and Wireless Propagation Letters, vol. 16, pp. 2332-2335, 2017.

[19] S. B. Venkatakrishnan, E. A. Alwan, and J. L. Volakis, "Multiband multi-beam performance evaluation of on-site coding digital beamformer using ultra-wideband antenna array," in 2017 XXXIInd General Assembly and Scientific Symposium of the International Union of Radio Science (URSI GASS), 2017, pp. 1-4.

[20] S. Bojja Venkatakrishnan, E. A. Alwan, and J. L. Volakis, "Wideband RF Self-Interference Cancellation Circuit for Phased Array Simultaneous Transmit and Receive Systems," IEEE Access, vol. 6, pp. 34253432, 2018.

[21] K. L. Scherer, S. J. Watt, E. A. Alwan, A. A. Akhiyat, B. Dupaix, W. Khalil, and J. L. Volakis, "Simultaneous transmit and receive system architecture with four stages of cancellation," in 2015 IEEE International Symposium on Antennas and Propagation USNC/URSI National Radio Science Meeting, 2015, pp. 520-521.

[22] X. Gu, D. Liu, C. Baks, O. Tageman, B. Sadhu, J. Hallin, L. Rexberg, P. Parida, Y. Kwark, and A. Valdes-Garcia, "Development, Implementation, and Characterization of a 64-Element Dual-Polarized PhasedArray Antenna Module for 28-GHz High-Speed Data Communica- tions," IEEE Transactions on Microwave Theory and Techniques, vol. 67, no. 7, pp. 2975-2984, July 2019.

[23] H. Kim, B. Park, S. Song, T. Moon, S. Kim, J. Kim, J. Chang, and Y. Ho, "A 28-GHz CMOS Direct Conversion Transceiver With Packaged $2 \times 4$ Antenna Array for 5G Cellular System," IEEE Journal of Solid-State Circuits, vol. 53, no. 5, pp. 1245-1259, 2018.

[24] B. Sadhu, Y. Tousi, J. Hallin, S. Sahl, S. Reynolds, O. Renstrm, K. Sjgren, O. Haapalahti, N. Mazor, B. Bokinge, G. Weibull, H. Bengtsson, A. Carlinger, E. Westesson, J. Thillberg, L. Rexberg, M. Yeck, X. Gu, D. Friedman, and A. Valdes-Garcia, "7.2 A 28GHz 32-element phasedarray transceiver IC with concurrent dual polarized beams and 1.4 degree beam-steering resolution for 5G communication," in 2017 IEEE International Solid-State Circuits Conference (ISSCC), 2017, pp. 128129.

[25] K. Kibaroglu, M. Sayginer, and G. M. Rebeiz, "A 28 GHz transceiver chip for 5G beamforming data links in SiGe BiCMOS," in 2017 IEEE Bipolar/BiCMOS Circuits and Technology Meeting (BCTM), 2017, pp. 74-77.

[26] J. Curtis, Hongyu Zhou, and F. Aryanfar, "A Fully Integrated Ka-Band Front End for 5G Transceiver," in 2016 IEEE MTT-S International Microwave Symposium (IMS), 2016, pp. 1-3.

[27] E. Westberg, J. Staudinger, J. Annes, and V. Shilimkar, "5G Infrastructure RF Solutions: Challenges and Opportunities," IEEE Microwave Magazine, vol. 20, no. 12, pp. 51-58, 2019.

[28] A. Watanabe, T. Lin, P. M. Raj, V. Sundaram, M. M. Tentzeris, R. R. Tummala, and T. Ogawa, "Leading-Edge and Ultra-Thin 3D Glass-Polymer 5G Modules with Seamless Antenna-to-Transceiver Signal Transmissions," in 2018 IEEE 68th Electronic Components and Technology Conference (ECTC), 2018, pp. 2026-2031.

[29] C. S. Geyik, Y. S. Mekonnen, Z. Zhang, and K. Aygn, "Impact of Use Conditions on Dielectric and Conductor Material Models for HighSpeed Package Interconnects," IEEE Transactions on Components, Packaging and Manufacturing Technology, vol. 9, no. 10, pp. 19421951, 2019.

[30] S. Sivapurapu, R. Chen, C. Mehta, Y. Zhou, M. L. F. Bellaredj, X. Jia, P. A. Kohl, T. Huang, S. K. Sitaraman, and M. Swaminathan, "MultiPhysics Modeling and Characterization of Components on Flexible Substrates," IEEE Transactions on Components, Packaging and Manufacturing Technology, vol. 9, no. 9, pp. 1730-1740, 2019.

[31] N. C. Wang, S. Sinha, B. Cline, C. D. English, G. Yeric, and E. Pop, "Replacing copper interconnects with graphene at a 7-nm node," in 2017 IEEE International Interconnect Technology Conference (IITC), 2017, pp. 1-3.

[32] Z. Cheng, W. Zhao, D. Wang, J. Wang, L. Dong, G. Wang, and W. Yin, "Analysis of cu-graphene interconnects," IEEE Access, vol. 6, pp. 53 499-53 508, 2018.

[33] Y. Wang, A. O. Watanabe, N. Ogura, P. M. Raj, and R. Tummala, "Sintered Nanocopper Paste for High-Performance 3D Heterogeneous Package Integration," Journal of Electronic Materials, pp. 1-9, 2020.

[34] T. Lin, P. M. Raj, A. Watanabe, V. Sundaram, R. Tummala, and M. M. Tentzeris, "Nanostructured miniaturized artificial magnetic conductors (amc) for high-performance antennas in $5 \mathrm{~g}$, iot, and smart skin applications," in 2017 IEEE 17th International Conference on Nanotechnology (IEEE-NANO), 2017, pp. 911-915.

[35] J. Lee and D. F. Sievenpiper, "Method for extracting the effective tensor surface impedance function from nonuniform, anisotropic, conductive patterns," IEEE Transactions on Antennas and Propagation, vol. 67, no. 5, pp. 3171-3177, 2019

[36] S. Hwangbo, A. Rahimi, and Y. Yoon, "Cu/co multilayer-based high signal integrity and low rf loss conductors for $5 \mathrm{~g} /$ millimeter wave applications," IEEE Transactions on Microwave Theory and Techniques, vol. 66, no. 8, pp. 3773-3780, 2018.

[37] "Advanced substrates: from FO to PCB," Yole Development, Tech. Rep., April 2017. [Online]. Available: t.ly/jNMt

[38] S. K. Kim, D. S. Oh, S. Hwang, B. W. Lee, S. Y. Cha, and T. H. Kim, "Electrical and Thermal Co-Analysis of Thermally Efficient SiP for High Performance Applications," in 2019 Electrical Design of Advanced Packaging and Systems (EDAPS), 2019, pp. 1-3.

[39] C. Chen, D. Yu, and L. Wan, "Electrical, Thermal and Mechanical Simulation for Embedded Silicon Fan-out Wafer Level Packaging," in 2018 IEEE International Conference on Integrated Circuits, Technologies and Applications (ICTA), 2018, pp. 158-159.

[40] J. Yook, D. Kim, B. Park, S. Sim, Y. Eo, and J. C. Kim, "A Compact 28 GHz RF Front-end Module using IPDs and Wafer-level Metal Fan-out Packaging," in 2019 49th European Microwave Conference (EuMC), 2019, pp. 436-439. 
[41] T. Hwang, D. Oh, J. Kim, E. Song, T. Kim, K. Kim, J. Lee, and T. Kim, "The Thermal Dissipation Characteristics of The Novel System-InPackage Technology (ICE-SiP) for Mobile and 3D High-end Packages," in 2019 IEEE 69th Electronic Components and Technology Conference (ECTC), 2019, pp. 614-619.

[42] S. You, S. Jeon, D. Oh, K. Kim, J. Kim, S. Cha, and G. Kim, "Advanced Fan-Out Package SI/PI/Thermal Performance Analysis of Novel RDL Packages," in 2018 IEEE 68th Electronic Components and Technology Conference (ECTC), 2018, pp. 1295-1301.

[43] Y. P. Zhang and D. Liu, "Antenna-on-Chip and Antenna-in-Package Solutions to Highly Integrated Millimeter-Wave Devices for Wireless Communications," IEEE Transactions on Antennas and Propagation, vol. 57, no. 10, pp. 2830-2841, 2009.

[44] F. X. Che, K. Yamamoto, V. S. Rao, and V. N. Sekhar, "Panel Warpage of Fan-Out Panel-Level Packaging Using RDL-First Technology," IEEE Transactions on Components, Packaging and Manufacturing Technology, vol. 10, no. 2, pp. 304-313, 2020.

[45] S. Ravichandran, S. Yamada, T. Ogawa, T. Shi, F. Liu, V. Smet, V. Sundaram, and R. Tummala, "Design and Demonstration of Glass Panel Embedding for 3D System Packages for Heterogeneous Integration Applications," Journal of Microelectronics and Electronic Packaging, vol. 16, no. 3, pp. 124-135, 2019. [Online]. Available: https://doi.org/10.4071/imaps.930748

[46] K. Hayashi, N. Kidera, and Y. Sato, "Low-Loss Glass Substrates Formulated with a Variety of Dielectric Characteristics for MillimeterWave Applications," in 2019 IEEE 69th Electronic Components and Technology Conference (ECTC), 2019, pp. 712-717.

[47] L. Martin, J. Matthias, B. T. Gore, W. J. Kozlovsky, R. Premerlani, A. Bruderer, M. Martina, T. Gottwald, T. Onishi, S. Onitake, S. Ravichandran, H. Maune, and M. Mydlak, "Attenuation of high Frequency Signals in Structured Metallization on Glass: Comparing Different Metallization Techniques with $24 \mathrm{GHz}, 77 \mathrm{GHz}$ and $100 \mathrm{GHz}$ Structures," in 2019 IEEE 69th Electronic Components and Technology Conference (ECTC), 2019, pp. 726-732.

[48] R. Bowrothu, S. Hwangbo, T. Schumann, and Y. Yoon, " $28 \mathrm{GHz}$ Through Glass Via (TGV) Based Band Pass Filter Using Through Fused Silica Via (TFV) Technology," in 2019 IEEE 69th Electronic Components and Technology Conference (ECTC), 2019, pp. 695-699.

[49] H. Xia, T. Zhang, L. Li, and F. Zheng, "A $1 \times 2$ Taper Slot Antenna Array With Flip-Chip Interconnect via Glass-IPD Technology for 60 GHz Radar Sensors," IEEE Access, vol. 8, pp. 61 790-61 796, 2020.

[50] A. O. Watanabe, H. Ito, R. P. Markondeya, R. R. Tummala, and M. Swaminathan, "Low-Loss Impedance-Matched Sub-25- $\mu \mathrm{m}$ Vias in 3-D Millimeter-Wave Packages," IEEE Transactions on Components, Packaging and Manufacturing Technology, vol. 10, no. 5, pp. 870-877, 2020.

[51] H. Ito, K. Kanno, A. Watanabe, R. Tsuyuki, R. Tatara, M. Raj, and R. R. Tummala, "Advanced Low-Loss and High-Density Photosensitive Dielectric Material for RF/Millimeter-Wave Applications," in 2019 International Wafer Level Packaging Conference (IWLPC), 2019, pp. $1-6$.

[52] C. O'Connor, R. Barr, J. Calvert, A. Choubey, M. Gallagher, E. Iagodkine, J.-S. Oh, A. Politis, and K. Wang, "Photopatternable Laminate BCB Dielectric," International Symposium on Microelectronics, vol. 2014, no. 1, pp. 000 580-000 584, 2014. [Online]. Available: https://doi.org/10.4071/isom-Poster6

[53] M. Tomikawa, H. Araki, Y. Kiuchi, and A. Shimada, "Low Dispersion Loss Polyimides for High Frequency Applications," in 2018 IEEE CPMT Symposium Japan (ICSJ), 2018, pp. 167-170.

[54] L. Maloratsky, Passive RF and Microwave Integrated Circuits. Elsevier Science, 2003. [Online]. Available: https://books.google.com/ books?id=jNa48HwPxQIC

[55] L. G. Maloratsky, "Design and Technology Tradeoffs in Passive RF and Microwave Integrated Circuits," High Frequency Electronics, pp. $40-54,2010$

[56] Z. Wu, "Design and demonstration of ultra-miniaturized glass-based 3d IPD diplexers and 3d IPAC RF front-end modules for LTE applications," Thesis, Georgia Institute of Technology, 2017. [Online]. Available: http://hdl.handle.net/1853/60702

[57] L. Miller, "RF Filter Technologies: Qorvo," Qorvo, e-Book, 2015. [Online]. Available: https://www.rfmw.com/data/qorvo_rf_filter_ technologies.pdf

[58] P. Matthews, "Approaching the 5G mmWave Filter Challenge - Microwave Journal," May 2019 [Online]. Available: https://www.microwavejournal.com/articles/ 32228-approaching-the-5g-mmwave-filter-challenge
[59] K. Ahn and I. Yom, "A Ka-band multilayer LTCC 4-pole bandpass filter using dual-mode cavity resonators," in 2008 IEEE MTT-S International Microwave Symposium Digest, Conference Proceedings, pp. 12351238.

[60] J. Showail, M. Lahti, K. Kari, E. Arabi, P. Rantakari, I. Huhtinen, T. Vaha-Heikkila, and A. Shamim, "SIW Cavity Filters with Embedded Planar Resonators in LTCC Package for 5G Applications," in 2018 48th European Microwave Conference (EuMC), 2018, pp. 757-760.

[61] J. Showail, "System on Package (SoP) Millimeter Wave Filters for 5G Applications," Thesis, KAUST, 2018. [Online]. Available: -http://hdl.handle.net/10754/627917

[62] Q. Guo, X. Y. Zhang, L. Gao, Y. C. Li, and J. Chen, "Microwave and Millimeter-Wave LTCC Filters Using Discriminating Coupling for Mode Suppression," IEEE Transactions on Components, Packaging and Manufacturing Technology, vol. 6, no. 2, pp. 272-281, 2016.

[63] X. Chen and K. Wu, "Self-Packaged Millimeter-Wave Substrate Integrated Waveguide Filter With Asymmetric Frequency Response,' IEEE Transactions on Components, Packaging and Manufacturing Technology, vol. 2, no. 5, pp. 775-782, 2012.

[64] M. Sharifi Sorkherizi and A. A. Kishk, "Self-Packaged, Low-Loss, Planar Bandpass Filters for Millimeter-Wave Application Based on Printed Gap Waveguide Technology," IEEE Transactions on Components, Packaging and Manufacturing Technology, vol. 7, no. 9, pp. 1419-1431, 2017.

[65] Y. Yan, Y. Chang, H. Wang, R. Wu, and C. H. Chen, "Highly Selective Microstrip Bandpass Filters in Ka-Band," in 2002 32nd European Microwave Conference, 2002, pp. 1-4.

[66] C. Tsai, J. Hsieh, W. Lin, L. Yen, J. Hung, T. Peng, H. Wang, C. Kuo, I. Huang, W. Chu, Y. Lei, C. H. Yu, L. C. Sheu, C. Hsieh, C. S. Liu, K. Yee, C. Wang, and D. Yu, "High performance passive devices for millimeter wave system integration on integrated fan-out (InFO) wafer level packaging technology," in 2015 IEEE International Electron Devices Meeting (IEDM), 2015, pp. 25.2.1-25.2.4.

[67] C. Hsu, C. Tsai, J. Hsieh, K. Yee, C. Wang, and D. Yu, "High Performance Chip-Partitioned Millimeter Wave Passive Devices on Smooth and Fine Pitch InFO RDL," in 2017 IEEE 67th Electronic Components and Technology Conference (ECTC), 2017, pp. 254-259.

[68] M. Ali, F. Liu, A. Watanabe, P. M. Raj, V. Sundaram, M. M. Tentzeris, and R. R. Tummala, "Miniaturized High-Performance Filters for 5G Small-Cell Applications," in 2018 IEEE 68th Electronic Components and Technology Conference (ECTC), May 2018, pp. 1068-1075.

[69] —-, "First Demonstration of Compact, Ultra-Thin Low-Pass and Bandpass Filters for 5G Small-Cell Applications," IEEE Microwave and Wireless Components Letters, vol. 28, no. 12, pp. 1110-1112, 2018.

[70] A. O. Watanabe, M. Ali, B. Tehrani, J. Hester, H. Matsuura, T. Ogawa, P. M. Raj, V. Sundaram, M. M. Tentzeris, and R. R. Tummala, "First Demonstration of $28 \mathrm{GHz}$ and $39 \mathrm{GHz}$ Transmission Lines and Antennas on Glass Substrates for 5G Modules," in 2017 IEEE 67th Electronic Components and Technology Conference (ECTC), 2017, pp. 236-241.

[71] M. Ali, A. Watanabe, T. Kakutani, P. M. Raj, R. R. Tummala, and M. Swaminathan, "Heterogeneous Integration of $5 \mathrm{G}$ and MillimeterWave Diplexers with 3D Glass Substrates," in 2020 IEEE 70th Electronic Components and Technology Conference (ECTC), to be published 2020.

[72] T. Martin, A. Ghiotto, T. Vuong, F. Lotz, and P. Monteil, "High performance air-filled substrate integrated waveguide filter post-process tuning using capacitive post," in 2017 IEEE MTT-S International Microwave Symposium (IMS), 2017, pp. 196-199.

[73] "Multilayer Band Pass Filter For 27.5-29.5GHz MMCB2528G5T0001A3," TDK, Datasheet, October 2019. [Online]. Available: t.ly/eGVD

[74] "28 GHz Surface Mount Bandpass Filter," Knowles Precison Devices, Datasheet, June 2019. [Online]. Available: https://www.knowlescapacitors.com/getattachment/5G-Filters/ 28GHz-Filters/B280MC1S_Datasheet.pdf

[75] "26 GHz Surface Mount Bandpass Filter," Knowles Precison Devices, Datasheet, July 2019. [Online]. Available: https://www.knowlescapacitors.com/getattachment/5G-Filters/ 26GHz-Filters/B259MC1S_Datasheet.pdf

[76] "38.5 GHz Surface Mount Bandpass Filter," Knowles Precison Devices, Datasheet, March 2018. [Online]. Available: https://www.knowlescapacitors.com/getattachment/Products/ Microwave-Products/Bandpass-Filters/B385MD0S_Datasheet.pdf

[77] Pasternack, "9 section bandpass filter with female SMA connectors, 27.5 to $31 \mathrm{GHz}$," Datasheet, 2019. [Online]. Available: https: //www.pasternack.com/images/ProductPDF/PE8747.pdf 
[78] A. Gupta and R. K. Jha, "A Survey of 5G Network: Architecture and Emerging Technologies," IEEE Access, vol. 3, pp. 1206-1232, 2015.

[79] D. Liu, X. Gu, C. W. Baks, and A. Valdes-Garcia, "An ApertureCoupled Dual-Polarized Stacked Patch Antenna for Multi-Layer Organic Package Integration," in 2019 IEEE International Symposium on Antennas and Propagation and USNC-URSI Radio Science Meeting, 2019, pp. 277-278.

[80] S. Shahramian, M. J. Holyoak, and Y. Baeyens, "A 16-element W-band phased array transceiver chipset with flip-chip PCB integrated antennas for multi-gigabit data links," in 2015 IEEE Radio Frequency Integrated Circuits Symposium (RFIC), 2015, pp. 27-30.

[81] K. Kibaroglu, M. Sayginer, and G. M. Rebeiz, "A scalable 64-element $28 \mathrm{ghz}$ phased-array transceiver with $50 \mathrm{dbm}$ eirp and 8-12 gbps $5 \mathrm{~g}$ link at 300 meters without any calibration," in 2018 IEEE/MTT S International Microwave Symposium - IMS, 2018, pp. 496-498.

[82] T. Thai, S. Dalmia, J. Hagn, P. Talebbeydokhti, and Y. Tsfati, "Novel Multicore PCB and Substrate Solutions for Ultra Broadband Dual Polarized Antennas for 5G Millimeter Wave Covering $28 \mathrm{GHz} \& 39 \mathrm{GHz}$ Range," in 2019 IEEE 69th Electronic Components and Technology Conference (ECTC), 2019, pp. 954-959.

[83] S. Li, H. T. Nguyen, T. Chi, C. Li, N. Cahoon, A. Kumar, G. Freeman, D. Harame, and H. Wang, "Performance of V-Band On-Chip Antennas in GlobalFoundries 45nm CMOS SOI Process for Mm-Wave 5G Applications," in 2018 IEEE/MTT-S International Microwave Symposium - IMS, 2018, pp. 1593-1596.

[84] J. D. Dunworth, A. Homayoun, B. Ku, Y. Ou, K. Chakraborty, G. Liu, T. Segoria, J. Lerdworatawee, J. W. Park, H. Park, H. Hedayati, D. Lu, P. Monat, K. Douglas, and V. Aparin, "A 28GHz Bulk-CMOS dualpolarization phased-array transceiver with 24 channels for $5 \mathrm{G}$ user and basestation equipment," in 2018 IEEE International Solid - State Circuits Conference - (ISSCC), 2018, pp. 70-72.

[85] A. Rashidian, S. Jafarlou, A. Tomkins, K. Law, M. Tazlauanu, and K. Hayashi, "Compact $60 \mathrm{GHz}$ Phased-Array Antennas With Enhanced Radiation Properties in Flip-Chip BGA Packages," IEEE Transactions on Antennas and Propagation, vol. 67, no. 3, pp. 1605-1619, 2019.

[86] X. Gu, D. Liu, C. Baks, O. Tageman, B. Sadhu, J. Hallin, L. Rexberg, and A. Valdes-Garcia, "A multilayer organic package with 64 dualpolarized antennas for $28 \mathrm{GHz} 5 \mathrm{G}$ communication," in 2017 IEEE MTT S International Microwave Symposium (IMS), 2017, pp. 1899-1901.

[87] A. Nafe, M. Sayginer, K. Kibaroglu, and G. M. Rebeiz, "2x64 DualPolarized Dual-Beam Single-Aperture $28 \mathrm{GHz}$ Phased Array with High Cross-Polarization Rejection for 5G Polarization MIMO," in 2019 IEEE MTT-S International Microwave Symposium (IMS), 2019, pp. 484-487.

[88] C. H. Tsai, C. W. Hsu, K. Y. Kao, T. C. Tang, C. L. Lu, K. C. Wu, H. P. $\mathrm{Pu}, \mathrm{K}$. Y. Lin, H. Wang, T. L. Wu, C. S. Liu, C. T. Wang, and D. C. H. Yu, "Fabrication and Characterization of Millimeter Wave 3D InFO Dipole Antenna Array Integrated with CMOS Front-end Circuits," in 2019 IEEE International Electron Devices Meeting (IEDM), 2019, pp. 25.3.1-25.3.4.

[89] F. Ahmed, M. Furqan, and A. Stelzer, " $120-\mathrm{GHz}$ and $240-\mathrm{GHz}$ Broadband Bow-Tie Antennas in eWLB Package for High Resolution Radar Applications," in 2018 48th European Microwave Conference (EuMC), 2018, pp. 1109-1112.

[90] A. Watanabe, M. Ali, R. Zhang, S. Ravichandran, T. Kakutani, P. M. Raj, R. Tummala, and M. Swaminathan, "Glass-Based IC-Embedded Antenna-Integrated Packages for 28-GHz High-Speed Data Communications," in 2020 IEEE 70th Electronic Components and Technology Conference (ECTC), 2020.

[91] A. Natarajan, S. K. Reynolds, M. Tsai, S. T. Nicolson, J. C. Zhan, D. G. Kam, D. Liu, Y. O. Huang, A. Valdes-Garcia, and B. A. Floyd, "A Fully-Integrated 16-Element Phased-Array Receiver in SiGe BiCMOS for 60-GHz Communications," IEEE Journal of Solid-State Circuits, vol. 46, no. 5, pp. 1059-1075, 2011.

[92] D. G. Kam, D. Liu, A. Natarajan, S. Reynolds, H. Chen, and B. A. Floyd, "LTCC Packages With Embedded Phased-Array Antennas for $60 \mathrm{GHz}$ Communications," IEEE Microwave and Wireless Components Letters, vol. 21, no. 3, pp. 142-144, 2011.

[93] W. Hong, A. Goudelev, K. Baek, V. Arkhipenkov, and J. Lee, "24Element Antenna-in-Package for Stationary 60-GHz Communication Scenarios," IEEE Antennas and Wireless Propagation Letters, vol. 10 , pp. 738-741, 2011.

[94] E. Cohen, M. Ruberto, M. Cohen, O. Degani, S. Ravid, and D. Ritter, "A CMOS Bidirectional 32-Element Phased-Array Transceiver at 60 GHz With LTCC Antenna," IEEE Transactions on Microwave Theory and Techniques, vol. 61, no. 3, pp. 1359-1375, 2013.
[95] T. Kamgaing, A. A. Elsherbini, T. W. Frank, S. N. Oster, and V. R. Rao, "Investigation of a photodefinable glass substrate for millimeterwave radios on package," in 2014 IEEE 64th Electronic Components and Technology Conference (ECTC), 2014, pp. 1610-1615.

[96] T. Zhang, Q. Lu, Z. Zhu, J. Hu, H. Xia, L. Li, and T. J. Cui, "Millimeterwave antenna-in-package applications based on d263t glass substrate," IEEE Access, vol. 8, pp. 67921-67928, 2020.

[97] M. Wojnowski, C. Wagner, R. Lachner, J. Bck, G. Sommer, and K. Pressel, "A 77-GHz SiGe single-chip four-channel transceiver module with integrated antennas in embedded wafer-level BGA package," in 2012 IEEE 62nd Electronic Components and Technology Conference, 2012, pp. 1027-1032.

[98] J. Bock and R. Lachner, "SiGe BiCMOS and eWLB packaging technologies for automotive radar solutions," in 2015 IEEE MTT$S$ International Conference on Microwaves for Intelligent Mobility (ICMIM), 2015, pp. 1-4.

[99] R. Hsieh, F. Chu, C. Ho, and C. Wang, "Advanced Thin-Profile FanOut with Beamforming Verification for 5G Wideband Antenna," in 2019 IEEE 69th Electronic Components and Technology Conference (ECTC), 2019, pp. 977-982.

[100] C. Tsai, J. Hsieh, M. Liu, E. Yeh, H. Chen, C. Hsiao, C. Chen, C. Liu, M. Lii, C. Wang, and D. Yu, "Array antenna integrated fanout wafer level packaging (InFO-WLP) for millimeter wave system applications," in 2013 IEEE International Electron Devices Meeting, 2013, pp. 25.1.1-25.1.4.

[101] N. Ogura, S. Ravichandran, T. Shi, A. Watanabe, S. Yamada, M. Kathaperumal, and R. Tummala, "First Demonstration of UltraThin Glass Panel Embedded (GPE) Package with Sheet Type Epoxy Molding Compound for 5G/mm-wave Applications," International Symposium on Microelectronics, vol. 2019, no. 1, pp. 000 202-7, 2019. [Online]. Available: https://doi.org/10.4071/2380-4505-2019.1.000202

[102] "6G Flagship." [Online]. Available: https://www.oulu.fi/6gflagship/

[103] "Key drivers and research challenges for 6G ubiquitous wireless intelligence," 6G Flagship - University of Oulu, White Paper, September 2019. [Online]. Available: http: //jultika.oulu.fi/files/isbn9789526223544.pdf

[104] A. Pouttu, "6Genesis-Taking the first steps towards 6G," in Proc. IEEE Conf. Standards Communications and Networking, 2018. [Online]. Available: cscn2018.ieeee-csen.org/files/2018/AriPouttu.pdf

[105] N. D. Tripathi and J. H. Reed, "Rohde \& Schwarz; 5G evolution - On the Path to 6G," Rohde \& Schwarz, White Paper, March 2020. [Online]. Available: https://www.mobilewirelesstesting.com/wp-content/uploads/2019/10/ 5G-evolution-on-the-path-to-6G-_wp_en_3608-3326-52_v0100.pdf

[106] Y. Yuan, Y. Zhao, B. Zong, and S. Parolari, "Potential key technologies for 6G mobile communications," Science China Information Sciences, vol. 63, no. 8, May 2020. [Online]. Available: http://dx.doi.org/10. 1007/s11432-019-2789-y

[107] W. Saad, M. Bennis, and M. Chen, "A Vision of 6G Wireless Systems: Applications, Trends, Technologies, and Open Research Problems," ArXiv, vol. abs/1902.10265, 2019.

[108] E. Yaacoub and M. Alouini, "A Key 6G Challenge and OpportunityConnecting the Base of the Pyramid: A Survey on Rural Connectivity," Proceedings of the IEEE, vol. 108, no. 4, pp. 533-582, 2020.

[109] H. Song, "Packages for Terahertz Electronics," Proceedings of the IEEE, vol. 105, no. 6, pp. 1121-1138, 2017. 